\title{
On the justification of the quasistationary approximation of several parabolic moving boundary problems - Part II
}

\author{
FRIEDRICH LIPPOTH \\ Institute of Applied Mathematics, Leibniz University of Hanover, Welfengarten 1, \\ 30167 Hannover, Germany \\ E-mail: lippoth@ifam.uni-hannover.de
}

[Received 17 September 2015 and in revised form 2 May 2016]

\begin{abstract}
We rigorously justify the quasistationary approximations of two moving boundary problems. We work out a systematic procedure to derive a priori estimates that allow to pass to the singular limit. The problems under our consideration are a one-phase osmosis model and the one-phase Stefan problem with Gibbs-Thomson correction and kinetic undercooling.
\end{abstract}

2010 Mathematics Subject Classification: Primary 35R37; Secondary 35B45, 93C70.

Keywords: Moving boundary problem, maximal regularity, quasistationary approximation, singular limit.

\section{Introduction}

This paper is the second part of work dealing with the question of strong convergence of classical solutions of parabolic moving boundary problems to a solution of an associated quasistationary model appearing as a singular limit of the full system. In a first part [11] we have considered an abstract system of nonlinear (and nonlinearly coupled) evolution equations evolving related to different time scales. We have studied the behaviour of solutions as the ratio of these scales becomes singular, and we have applied our abstract results to a moving boundary problem modelling the growth of an avascular tumor and to a quasilinear version of the Keller-Segel model on a bounded domain in $\mathbb{R}^{N}$.

However, the abstract results from the first part do not apply to a class of evolution problems which involve inhomogeneous and/or nonautonomous (possibly also nonlinear) side conditions that can hardly be hidden in a linear space serving as domain of definition for a leading (quasi-) linear operator. A famous example of such an evolution problem (after a suitable coordinate change) is the Stefan problem with Gibbs-Thomson correction (with or without kinetic undercooling).

Recently, the investigation of these kind of problems within the framework of analytic semigroups has been significantly progressed provided one focusses on systems that are in an appropriate sense parabolic [6], [12], [13], [21]. The underlying philosophy is to solve simultaneously for all unknowns.

In its generality (cf. [6]), this approach is not designed to measure the influence of different speed parameters on the evolution of different unknowns. In order to do so, considering a fast evolving quantity (which is therefore in a quasistationary state relative to the others) as a function of the other unknowns turns out to be a fruitful ansatz.

In this paper we systematically work out this idea. Our strategy will be applied to the one-phase Stefan problem with Gibbs-Thomson correction and kinetic undercooling as well as to a one-phase 
model of osmotic cell swelling. We will prove local existence of classical solutions of the (speed) parameter dependent full problems on a uniform interval of existence and rigorously establish strong convergence of these solutions to the solution of the corresponding quasistationary problem. Beside parabolicity, the following structural properties of the problems under consideration are essential ingredients of the proof: in the case of the osmosis problem we make use of solute conservation, while in the case of the Stefan problem with kinetic undercooling spectral properties of the RobinLaplacian are important cf. Section 5 (Conclusions). This paper is organized as follows:

Section 2: we introduce the models we are going to consider. Section 3 is devoted to the osmosis model: Section 3.1: we construct the abstract framework that carries our analysis. Section 3.2: we derive a priori estimates for a parameter dependent nonlinear parabolic problem which are uniform w.r.t. a certain class of data (consisting of coefficient functions, right hand sides etc.). Section 3.3: we prove existence of solutions of the full nonlinear parameter dependent problem on a short interval of existence not depending on the parameter by means of a fixed point argument. Section 3.4: we prove the convergence of these solutions. Section 4: we sketch how our approach works for the Stefan problem. Section 5 contains a short summary of the essential ingredients of our analysis. Section 6 collects necessary technical material and contains some outstanding proofs.

The justification of quasistationary approximations has already been considered by other authors. In [9], [23] V. A. Solonnikov and E. V. Frolova prove the convergence of classical solutions of the Navier-Stokes free boundary problem to the Stokes system with free boundary (the case of 'infinite kinematic viscosity') and of classical solutions of the classical Stefan problem (i.e. without Gibbs-Thomson correction and without kinetic undercooling) to the Hele-Shaw problem. The methods used there are not based on semigroup theory and thus are of different nature than our ones. However, in the case of the Stefan problem Frolova/Solonnikov arrive at a convergence rate of order $\odot\left(e^{-c t / \varepsilon)}\right)(+\odot(\varepsilon))$ which coincides with our findings (cf. Eq. (4.10), also Lemmas 2.1-2.3 in [11]). Observe that it is natural to expect a singular behaviour at $t=0$ since the initial configurations of a 'full' problem and its quasistationary limit in the bulk phase are different in general, cf. Remark 3.3 in [11]. The Stefan problem is also considered in [27].

\section{The models}

\subsection{Osmosis in a resting solvent}

We consider the one-phase version of a moving boundary problem modelling osmosis:

$$
\begin{aligned}
& \varepsilon \partial_{t} v-\Delta v=0 \quad \text { in } \Omega(t), \\
& \partial_{n} v+\varepsilon v V_{n}=0 \quad \text { on } \Gamma(t), \\
& V_{n}=H+v \quad \text { on } \Gamma(t),
\end{aligned}
$$

or, equivalently,

$$
\left.\begin{array}{rlrl}
\varepsilon \partial_{t} v-\Delta v & =0 & & \text { in } \Omega(t), \\
\partial_{n} v+\varepsilon\left[H v+v^{2}\right] & =0 & & \text { on } \Gamma(t), \\
V_{n} & =H+v & \text { on } \Gamma(t),
\end{array}\right\}
$$

$v(0):=v_{0}, \Gamma(0):=\Gamma_{0}$. The semipermeable membrane $\Gamma(t)$ moves freely in an incompressible fluid at rest. The membrane encloses a region $\Omega(t) \subset \mathbb{R}^{N}$, where a certain amount of a solute is dissolved. Its concentration at position $x \in \Omega(t)$ and at time $t$ is denoted by $v=v(t, x)$. $H=H(t, x)$ is the $((N-1)$ - fold) mean curvature of the surface $\Gamma(t)$ at $x \in \Gamma(t)$ (taken negative 
where $\Omega(t)$ is convex), and $V_{n}=V_{n}(t, x)$ denotes the normal Velocity of the family $\{\Gamma(t)\}$ at $x \in \Gamma(t)$.

The equations in (2.2) are already in dimensionless form. The positive number $\varepsilon$ is a dimensionless parameter which cannot be scaled out of the system. It can be interpreted as the ratio of the typical time scales of relaxation of the membrane without solute and of diffusion of solute inside the membrane. Thus, the singular limit $\varepsilon=0$ corresponds to the case of 'infinite fast diffusion', which may be called a quasistationary approximation. More details about the modelling of the system (2.2) can be found in [12], [14], [19], [24], [25], [28].

The model (2.2) has already been considered in [12], where local existence and uniqueness of classical solutions has been proved. Moreover, close to equilibria, solutions have been constructed that live on arbitrary long (but not infinite) time intervals. In [13] the latter result has been sharpened by a rigorous proof of local attractivity of the manifold of equilibrium solutions for a two-phase version of (2.2).

At this point we want to work out a more concise formulation of the limit case $\varepsilon=0$ of (2.2): Observe that for any $\varepsilon>0$ the total amount of solute is a preserved quantity in the model (2.2). Indeed, using the transport and divergence theorem, we see that any classical solution $\left(v_{\varepsilon}, \Gamma_{\varepsilon}\right)$ of suitable regularity satisfies

$$
\frac{d}{d t} \int_{\Omega_{\varepsilon}(t)} v_{\varepsilon}(t)=\int_{\Omega_{\varepsilon}(t)} \dot{v}_{\varepsilon} d x+\int_{\Gamma_{\varepsilon}} v_{\varepsilon}(t) \cdot V_{n, \varepsilon}(t) d \sigma_{\varepsilon}(x)=0
$$

where $d \sigma_{\varepsilon}$ is the surface measure of $\Gamma_{\varepsilon}$. Thus $\int_{\Omega_{\varepsilon}(t)} v_{\varepsilon}(t)=\int_{\Omega_{0}} v_{0}$. Formally, in the limit case $\varepsilon=0$ the system (2.2) reduces to

$$
\left.\begin{array}{rlrl}
\Delta v & =0 & & \text { in } \tilde{\Omega}(t), \\
\partial_{n} v & =0 & & \text { on } \tilde{\Gamma}(t), \\
V_{n} & =H+v & & \text { on } \tilde{\Gamma}(t),
\end{array}\right\}
$$

$\tilde{\Gamma}(0)=\Gamma_{0}$. Thus we see that any classical solution $(v, \tilde{\Gamma})$ of $(2.4)$ has the property that $v$ is constant in the space variable, i.e. $v(t, x)=\tilde{\omega}(t)$. Taking the convergence $v_{\varepsilon} \stackrel{\varepsilon \rightarrow 0}{\longrightarrow} \tilde{\omega}, \Omega_{\varepsilon} \stackrel{\varepsilon \rightarrow 0}{\longrightarrow} \tilde{\Omega}$ for granted in a strong enough sense, by the conservation of solute, this constant is given by

$$
\tilde{\omega}(t)=\int_{\Omega_{0}} v_{0} / \operatorname{vol}(\tilde{\Omega}(t)) \quad=: \quad M_{0} / \operatorname{vol}(\tilde{\Omega}(t))
$$

and (2.4) reduces to

$$
V_{n}=H+\tilde{\omega} \quad \text { on } \tilde{\Gamma}(t), \quad \tilde{\Gamma}(0)=\Gamma_{0},
$$

which can be viewed as a mean curvature flow including a 'stopping mechanism'. Some of its properties (including local existence of classical solutions) have been investigated in [26].

Observe that studying the quasistationary approximation at first instead of a full problem is a convenient strategy in order to understand analytic properties of solutions of moving boundary problems, because, at least from a mathematical point of view, the latter problems are fairly more involved in general. For the osmosis problem, there is an extensive list of questions that probably could be answered for the fast diffusion limit, but that seem out of reach when considering the complete system (2.2), such as investigating geometric properties of the moving domain, analysing an intriguing variational structure (cf. [14], [29], [30]). 
2.2 The one-phase Stefan problem with Gibbs-Thomson correction and kinetic undercooling

The Stefan problem describes the phase transition in a system consisting of a liquid and a solid phase. In the one-phase version one looks for a temperature distribution $v$ (say in the liquid phase $\Omega(t))$ and a moving interface $\Gamma(t):=\partial \Omega(t)$ satisfying

$$
\begin{aligned}
& \varepsilon \partial_{t} v-\Delta v=0 \quad \text { in } \Omega(t), \\
& \partial_{n} v+V_{n}=0 \quad \text { on } \Gamma(t), \\
& V_{n}=H+v \text { on } \Gamma(t),
\end{aligned}
$$

or equivalently

$$
\left.\begin{array}{rlrl}
\varepsilon \partial_{t} v-\Delta v & =0 & & \text { in } \Omega(t), \\
\partial_{n} v+v & =-H & & \text { on } \Gamma(t), \\
V_{n} & =H+v & & \text { on } \Gamma(t),
\end{array}\right\}
$$

$v(0):=v_{0}, \Gamma(0):=\Gamma_{0}$. The temperature in the solid phase (surrounding $\bar{\Omega}(t)$ ) is assumed to be constant. The equations are in dimensionless form. We have scaled as many constants as possible to 1 . The remaining dimensionless parameter $\varepsilon$ contains information about capacity of heat per unit volume, thermal conductivity and relaxation of the interface. The formal limit obtained by setting $\varepsilon=0$ is the quasistationary one-phase Stefan problem with Gibbs-Thomson correction and kinetic undercooling. It describes the case of a low capacity of heat (relative to other parameters). Both, the limit and the full problem are considered in [10], the full problem is discussed in [21]. The Stefan problem has been considered by various authors for more then a century. For a mathematical discussion of the problem we refer to [10], [21] and the references given therein.

Due to the nonlinear boundary condition (the second equation in (2.2)), at least for the investigations we have in mind, the osmosis model (2.2) is a more challenging problem than the Stefan problem (4.1) in several aspects. Indeed, it turns out that large parts of the procedure used to prove a priori estimates for the osmosis problem can be applied to the Stefan problem (except some details we are going to collect in Section 4). On the other hand, the arguments used to finally establish our convergence result (Sections 3.4,4) can hardly be formulated in a 'problem independent way'. Therefore we refrain in this paper from deriving a general abstract framework as in [11].

\section{The one-phase osmosis model}

\subsection{The abstract setting}

We apply the direct mapping method in order to transform the system (2.2) to a set of equations over a fixed reference domain. We shall briefly sketch this well known procedure:

Throughout the article we keep the numbers $N \in \mathbb{N}, N \geqslant 2,0<\beta<\alpha<1$ fixed. From now on we shall focus on the model (2.2). We assume that

(I1) $\Omega_{0} \subset \mathbb{R}^{N}$ is a bounded connected open set and $\Gamma_{0}:=\partial \Omega_{0}$ is a closed compact hypersurface of regularity class $h^{4+\alpha}$

(I2) For $\varepsilon>0, v_{0}^{\varepsilon} \in h^{2+\alpha}\left(\bar{\Omega}_{0}\right)$ satisfies

$\circ \partial_{n} v_{0}^{\varepsilon}+\varepsilon\left(H_{\Gamma_{0}} v_{0}^{\varepsilon}+\left(v_{0}^{\varepsilon}\right)^{2}\right)=0$ on $\Gamma_{0}$;

○ $v_{0}^{\varepsilon} \geqslant 0$ in $\bar{\Omega}_{0}$;

$\circ \sup _{\varepsilon>0}\left\|v_{0}^{\varepsilon}\right\|_{h^{2+\alpha}\left(\bar{\Omega}_{0}\right)}<\infty$;

$\circ$ the mapping $\varepsilon \mapsto \int_{\Omega_{0}} v_{0}^{\varepsilon}$ is constant. 
Here, $h^{m+\gamma}$ denotes the little Hölder space, see Section 6 (appendix). Observe that the condition (I2) is satisfied for $v_{0}^{\varepsilon}:=\tilde{v}_{0}(\varepsilon>0)$, where $\tilde{v}_{0}$ is a non-negative function in $\overline{\Phi\left(\Omega_{0}\right)} h^{2, \alpha}\left(\bar{\Omega}_{0}\right)$.

We want to use the direct mapping method to transform system (2.2) to a set of equations given over a fixed and smooth reference domain. The unknown family of surfaces $\{\Gamma(t)\}:=\{\partial \Omega(t)\}$ will be described by a signed distance function with respect to that surface. In order to do these transformations, we need some preparation:

Given any closed compact hypersurface $m \subset \mathbb{R}^{N}$ of class $C^{2}$, let $T_{\zeta}=T_{\zeta}(m)$ be an open tubular neighborhood of $m$, i.e. the diffeomorphic image of the mapping

$$
X_{m}: m \times(-\zeta, \zeta) \rightarrow \mathbb{R}^{N}, \quad(x, a) \mapsto x+a \cdot v_{m}(x),
$$

where $\nu_{\mathfrak{m}}(x)$ is the outer unit normal vector at $x \in \mathfrak{m}$ and $\zeta>0$ is sufficiently small. It is convenient to decompose the inverse of $X_{m}$ into $X_{m}^{-1}=\left(P_{m}, \Lambda_{m}\right)$, where $P_{m}(x)$ is the metric projection of a point $x \in T_{\zeta}$ onto $m$ and $\Lambda_{m}$ is the signed distance function with respect to $m$. Let

$$
\operatorname{Ad}_{m, \tau}:=\left\{\sigma \in C^{1}(m) ;\|\sigma\|_{C(m)}<\tau / 5\right\} \quad(\tau>0) .
$$

If $\tau>0$ is small enough, then the mapping $\theta_{\sigma}(x):=x+\sigma(x) \cdot \nu_{m}(x)$ is for each $\sigma \in \operatorname{Ad}_{m, \tau}$ a diffeomorphism mapping $m$ onto $m_{\sigma}:=\theta_{\sigma}[m]$.

Due to Theorem 4.2 in [4] we can fix a number $\zeta>0$ and a triple $\left(\Omega, S_{\zeta}(\Gamma), \rho_{0}\right)$ in the following way:

- $\Omega \subset \Omega_{0}$ is a domain and $\Gamma:=\partial \Omega$ is a closed compact real analytic hypersurface;

- $S:=S_{\zeta}(\Gamma)$ is an open tubular neighborhood of $\Gamma, \Gamma_{0} \subset S$;

- $\rho_{0} \in h^{4+\alpha}(\Gamma) \cap \operatorname{Ad}_{\Gamma, \zeta}$ and the mapping $\theta_{\rho_{0}}: \Gamma \rightarrow \Gamma_{0}$ is a $h^{4+\alpha}$-diffeomorphism. In particular, $\Gamma_{0}=\Gamma_{\rho_{0}}$.

From now on let $\zeta>0,\left(\Omega, S, \rho_{0}\right)$ be chosen as described above and let $A d:=A d_{\Gamma, \zeta}$.

Observe that $\theta_{\sigma}[\Gamma] \subset S$ for all $\sigma \in \operatorname{Ad}$. Suppose that $\sigma \in \operatorname{Ad} \cap h^{m+\gamma}(\Gamma)$ for some $(m, \gamma) \in \mathbb{N} \times$ $(0,1)$. It is not difficult to see that then $\theta_{\sigma} \in h^{m+\gamma}\left(\Gamma, \mathbb{R}^{N}\right)$ and $\theta_{\sigma}^{-1} \in h^{m+\gamma}\left(\Gamma_{\sigma}, \mathbb{R}^{N}\right)$. Moreover, given $\sigma \in \operatorname{Ad} \cap h^{m+\gamma}(\Gamma)$, the mapping $\theta_{\sigma}$ extends to a diffeomorphism

$$
\theta_{\sigma} \in \operatorname{Diff}^{m, \gamma}\left(\mathbb{R}^{N}, \mathbb{R}^{N}\right),\left.\quad \theta_{\sigma}\right|_{\Omega} \in \operatorname{Diff}^{m, \gamma}\left(\Omega, \Omega_{\sigma}\right) \quad\left(\Omega_{\sigma}:=\theta_{\sigma}[\Omega]\right),
$$

such that we have $\partial \Omega_{\sigma}=\Gamma_{\sigma}$. Let $\tilde{\tau}:=\zeta / 5$ and $\chi \in C^{\infty}(\mathbb{R},[0,1])$ satisfy $\left.\chi\right|_{[-\tilde{\tau}, \tilde{\tau}]} \equiv 1$, $\left.\left.\chi\right|_{(-\infty,-3 \tilde{\tau}]} \equiv \chi\right|_{[3 \tilde{\tau}, \infty)} \equiv 0,\left\|\chi^{\prime}\right\|_{\infty}<1 / \tilde{\tau}$. Then the mapping

$$
y \mapsto\left\{\begin{array}{lll}
X_{\Gamma}\left(P_{\Gamma}(y), \Lambda_{\Gamma}(y)+\chi\left(\Lambda_{\Gamma}(y)\right) \cdot \sigma\left(P_{\Gamma}(y)\right)\right), & \text { if } \quad y \in S \\
y, & \text { if } y \notin S
\end{array}\right.
$$

( $\sigma \in \mathrm{Ad}$ ), again denoted by $\theta_{\sigma}$, is an appropriate extension, the so-called Hanzawa diffeomorphism. Note that for $\sigma \in$ Ad the surface $\Gamma_{\sigma}$ is the zero level set of the function $\varphi_{\sigma}$ defined by

$$
\varphi_{\sigma}(x)=\Lambda_{\Gamma}(x)-\sigma\left(P_{\Gamma}(x)\right),
$$

$x \in S$, i.e. $\Gamma_{\sigma}=\varphi_{\sigma}^{-1}[\{0\}]$. For later use we set

$$
L_{\sigma}(x):=\left|\nabla \varphi_{\sigma}\right|\left(\theta_{\sigma}(x)\right) .
$$


It can be shown that $L_{\sigma}>0$ on $\Gamma$ for all $\sigma \in$ Ad. Given $\sigma \in \operatorname{Ad}$, let $\theta_{\sigma}^{*}, \theta_{*}^{\sigma}$ denote the pull-back and push-forward operators induced by $\theta_{\sigma}$, i.e. $\theta_{\sigma}^{*} f=f \circ \theta_{\sigma}, \theta_{*}^{\sigma} g=g \circ \theta_{\sigma}^{-1}$. If suitable functions $b, \rho$ are time dependent, i.e. $b=b(t, x), \rho=\rho(t, x)$, we define $\left[\theta_{\rho}^{*} b\right](t, x):=\left[\theta_{\rho(t)}^{*} b(t, \cdot)\right](x)$, analogue for $\theta_{*}^{\rho}$.

Using this notation, for suitable $\rho$ we can introduce the transformed operators

$$
\begin{aligned}
A(\rho) u & :=\theta_{\rho}^{*}\left(\Delta\left(\theta_{*}^{\rho} u\right)\right) \\
B(\rho) u & :=\theta_{\rho}^{*}\left(\nabla\left(\theta_{*}^{\rho} u\right) \cdot \nabla \varphi_{\rho} /\left|\nabla \varphi_{\rho}\right|\right) \\
H(\rho) & :=\theta_{\rho}^{*} H_{\left[\Gamma_{\rho}\right] .}
\end{aligned}
$$

System (2.2) can be equivalently rewritten as

$$
\begin{aligned}
\varepsilon \partial_{t} u-A(\rho) u & =\varepsilon R(\rho, u) & & \text { in } \Omega \times(0, T], \\
B(\rho) u+\varepsilon\left[u H(\rho)+u^{2}\right] & =0 & & \text { on } \Gamma \times[0, T], \\
\partial_{t} \rho-L_{\rho} P(\rho) \rho & =L_{\rho} Q(\rho)+L_{\rho} u & & \text { on } \Gamma \times(0, T], \\
u(0) & =u_{0}^{\varepsilon} & & \text { in } \Omega, \\
\rho(0) & =\rho_{0} & & \text { on } \Gamma,
\end{aligned}
$$

where $u_{0}^{\varepsilon}:=\theta_{\rho_{0}}^{*} v_{0}^{\varepsilon}$. Here we used the splitting

$$
H(\rho)=P(\rho) \rho+Q(\rho),
$$

cf. [8] (the following is an easy way to understand the structure of the nonlinear operator $H(\rho)$ : let $\Delta_{\rho}$ be the Laplace-Beltrami operator of $\Gamma_{\rho}$ w.r.t the metric inherited from the ambient space $\mathbb{R}^{N}$. Let further $n_{\rho}$ be the outer unit normal field and $H_{\rho}$ be the mean curvature of $\Gamma_{\rho}$. Recall that

$$
H_{\rho}=\left(\Delta_{\rho} \xi_{\rho} \mid n_{\rho}\right)_{\mathbb{R}^{N}},
$$

where $\xi_{\rho}$ assigns to each point of $\Gamma_{\rho}$ its cartesian coordinates and $\Delta_{\rho}$ acts componentwise on $\xi_{\rho}$. Parameterizing $\Gamma_{\rho}$ over $\Gamma$ (i.e. letting $\xi_{\rho}(x):=x+\rho(x) \cdot n_{\Gamma}(x), x \in \Gamma$ ) we obtain in local coordinates on $\Gamma$

$$
\Delta_{\rho} \xi_{\rho}=\frac{1}{\sqrt{G_{\rho}}} \sum_{i, j=1}^{N-1} \partial_{i}\left(\sqrt{G_{\rho}} g_{\rho}^{i j} \partial_{j} \xi_{\rho}\right),
$$

where $g_{i j}^{\rho}=\left(\partial_{i} \xi_{\rho} \mid \partial_{j} \xi_{\rho}\right)_{\mathbb{R}^{N}},\left(g_{\rho}^{i j}\right)=\left(g_{i j}^{\rho}\right)^{-1}$ and $G_{\rho}=\operatorname{det}\left(g_{i j}^{\rho}\right)$. From equation (3.5) it is clear that $H(\rho)$ is of second order in $\rho$ and that it has a quasilinear structure).

The term $R$ arises from the transformation of the time derivative $v_{t}$ and is determined by

$$
R(w, \sigma)(y)=r_{0}\left(L_{\sigma}[H(\sigma)+w], B_{\mu}(\sigma) w\right)(y), \quad y \in \Omega,
$$

where $w \in C^{1}(\bar{D}), \sigma \in \operatorname{Ad} \cap C^{2}(\Gamma)$ and

$$
\begin{aligned}
& r_{0}(h, k)(y):= \begin{cases}\chi\left(\Lambda_{\Gamma}(y)\right) \cdot h\left(P_{\Gamma}(y)\right) \cdot k(y), & \text { if } \quad y \in \Omega \cap S \\
0, & \text { if } \quad y \in \Omega \backslash(\Omega \cap S),\end{cases} \\
& B_{\mu}(\sigma) v(y)=\theta_{\sigma}^{*} \nabla\left(\theta_{*}^{\sigma} v\right)(y) \cdot\left(v_{\Gamma} \circ P_{\Gamma}\right)(y), \quad y \in \Omega \cap S .
\end{aligned}
$$

The derivation of $R$ is a straightforward calculation, cf. [7], [10]. 
The transformed version of the mean curvature flow type equation (2.5) reads:

$$
\partial_{t} \rho-L_{\rho} P(\rho) \rho=L_{\rho} Q(\rho)+L_{\rho} \tilde{u}_{\rho}, \quad \rho(0)=\rho_{0}
$$

( $\rho_{0}$ as specified above), where

$$
\tilde{u}_{\rho}(t)=M_{0} / \operatorname{vol}\left(\Omega_{\rho(t)}\right)=M_{0} /\left(\int_{\Omega}\left|\operatorname{det} D \theta_{\rho(t)}\right| d x\right) .
$$

To state our main result (and for further analysis) we need to specify some function spaces, cf. also Section 6 (Appendix):

If $J \subset[0, \infty)$ is a perfect interval, let $\dot{J}:=J \backslash \partial J$ and

- $\mathbb{E}_{1}(J):=B U C\left(\dot{J}, h^{2+\alpha}(\bar{\Omega})\right) \cap B U C^{1}\left(\dot{J}, h^{\alpha}(\bar{\Omega})\right)$;

- $\mathbb{E}_{0}(J):=B U C\left(\dot{J}, h^{\alpha}(\bar{\Omega})\right)$;

- $\mathbb{F}(J):=B U C\left(\dot{J}, h^{1+\alpha}(\Gamma)\right) \cap h^{(1+\alpha) / 2}(\bar{J}, C(\Gamma))$;

- $\mathbb{G}(J):=\left\{(f, g, x) \in \mathbb{E}_{0}(J) \times \mathbb{F}(J) \times h^{2+\alpha}(\bar{\Omega}), B\left(\rho_{0}\right) x=g(0)\right\}$, $\rho_{0}$ as specified above. Finally, let

- $\mathbb{Y}(J):=B U C\left(\dot{J}, h^{4+\alpha}(\Gamma)\right) \cap B U C^{1}\left(\dot{J}, h^{2+\alpha}(\Gamma)\right.$.

If $X \hookrightarrow C(\bar{\Omega})$, we denote by $X_{+}$the closed subspace of nonnegative functions. If $J \subset \mathbb{R}$ and $\mathbb{X} \subset X^{J}$, let $\mathbb{X}_{+}:=\mathbb{X} \cap\left(X_{+}\right)^{J}$. Given $T>0$ let $J_{T}:=[0, T]$.

Theorem 3.1 Let $u_{0}^{\varepsilon}, \rho_{0}$ be as specified above. Then there exist $T^{*}, \varepsilon_{0}>0$ with the following properties:

- For each $0<\varepsilon<\varepsilon_{0}$ system (3.3) possesses a unique classical solution $\left(u_{\varepsilon}, \rho_{\varepsilon}\right) \in \mathbb{E}_{1}\left(J_{T^{*}}\right)_{+} \times$ $\mathbb{Y}\left(J_{T^{*}}\right)$.

- $\left(u_{\varepsilon}, \rho_{\varepsilon}\right)(t) \stackrel{\varepsilon \rightarrow 0}{\longrightarrow}\left(\tilde{u}_{\tilde{\rho}}, \tilde{\rho}\right)(t)$, where $\tilde{u}_{\tilde{\rho}}$ is determined by (3.8) and $\tilde{\rho} \in \mathbb{Y}\left(J_{T^{*}}\right)$ is the unique solution of (3.7).

The convergence takes place in $C\left(\left[\delta, T^{*}\right], h^{2+\beta}(\bar{\Omega})\right) \times C\left(\left[0, T^{*}\right], h^{4+\beta}(\Gamma)\right)$ for each $\delta \in\left(0, T^{*}\right)$.

\subsection{Estimates for $u(\rho)$}

Let $u_{0}^{\varepsilon}, \rho_{0}$ be as specified in Section 3.1. For $T>0, M>\left\|\rho_{0}\right\|_{h^{4+\beta}(\Gamma)}$ (to be determined later) and $J_{T}:=[0, T]$. Let

$$
\begin{aligned}
& V:=V_{M}\left(J_{T}\right) \\
& :=\left\{\rho \in C\left(J_{T}, h^{4+\beta}(\Gamma) \cap \mathrm{Ad}\right) ;\|\rho(t)\|_{h^{4+\beta}(\Gamma)} \leqslant M ;\|\rho(t)-\rho(s)\|_{h^{2+\beta}(\Gamma)} \leqslant M \cdot|t-s|\right\}
\end{aligned}
$$

and observe that the constant map $t \mapsto \rho_{0}$ belongs to $V_{M}\left(J_{T}\right)$. In this section we consider the problem

$$
\left.\begin{array}{rlrl}
\varepsilon \partial_{t} u-A(\rho) u & =\varepsilon R(\rho, u) & & \text { in } \Omega \times(0, T], \\
B(\rho) u & =-\varepsilon u H(\rho)-\varepsilon u^{2} & & \text { on } \Gamma \times[0, T], \\
u(0) & =u_{0}^{\varepsilon} & & \text { in } \Omega
\end{array}\right\}
$$

for a given $T>0$ and $\rho \in V_{M}\left(J_{T}\right)$. The latter set, of course, represents a 'uniformly bounded family of domains'. It has the following properties: 
Lemma 3.2 Let $T>0, M>\left\|\rho_{0}\right\|_{h^{4+\beta}(\Gamma)}$. Then $V_{M}\left(J_{T}\right)$ is a bounded closed convex subset of $C\left(J_{T}, h^{4+\beta}(\Gamma)\right)$.

Proof. This can be easily seen by similar arguments as in [11], Lemma 3.1.

Our goal in this section is to prove the following Lemma:

Lemma 3.3 Let $u_{0}^{\varepsilon}, \rho_{0}$ be as specified in Section 3.1. There is a number $C>0$ such that the following holds true: Given $M>\left\|\rho_{0}\right\|_{h^{4+\beta}(\Gamma)}$ there exists $T^{*}>0, \varepsilon_{0}>0$ such that for all $(T, \varepsilon) \in\left(0, T^{*}\right] \times\left(0, \varepsilon_{0}\right]$ and $\rho \in V_{M}\left(J_{T}\right)$ problem (3.10) possesses a unique classical nonnegative global solution $u=u(\rho, \varepsilon)$, i.e. a solution in the class $\mathbb{E}_{1}\left(J_{T}\right)_{+}$. Moreover,

$$
\|u(\rho, \varepsilon)\|_{C\left(J_{T}, h^{2+\alpha}(\bar{\Omega})\right)}+\varepsilon\left\|u_{t}(\rho, \varepsilon)\right\|_{C\left(J_{T}, h^{\alpha}(\bar{\Omega})\right)} \leqslant C,
$$

$(T, \varepsilon) \in\left(0, T^{*}\right] \times\left(0, \varepsilon_{0}\right], \rho \in V_{M}\left(J_{T}\right)$. For each fixed $(T, \varepsilon) \in\left(0, T^{*}\right] \times\left(0, \varepsilon_{0}\right]$ and $v \in(0, \alpha)$ the mapping

is continuous.

$$
\rho \mapsto u(\varepsilon, \rho): V_{M}\left(J_{T}\right) \rightarrow C\left(J_{T}, h^{2+v}(\bar{\Omega})\right)
$$

We informally sketch our strategy of proving Lemma 3.3: First we make sure that problem (3.10) indeed possesses a (nonnegative) classical solutions on some time interval possibly depending on $\varepsilon$ and $\rho$ (Lemma 3.4). Then we estimate the maximum of this solution uniformly in $\varepsilon>0$ and $\rho \in V_{M}\left(J_{T}\right)$ (Lemma 3.5). From this, we derive an estimate for the $L_{p}$-norm (in space and time) for the solution of a scaled problem associated to (3.10). Then we use the nonlinear structure of the problem to apply first the maximal $L_{p}$-regularity result 6.1 (i) and then bootstrap with the help of 6.1 (ii) to the estimate (3.11) which guarantees global existence by suitable abstract results (Lemma 3.6).

We shall also make repeatedly use of the fact (cf. (I2)) that $\sup \left\{\left\|u_{0}^{\varepsilon}\right\|_{h^{2+\alpha}(\bar{\Omega})} ; \varepsilon>0\right\}<\infty$. In order to keep things simple we write $u_{0}$ instead of $u_{0}^{\varepsilon}$.

Lemma 3.4 Let $\varepsilon>0, u_{0}$ be as specified. Let $T>0, M>\left\|\rho_{0}\right\|_{h^{4+\beta}(\Gamma)}, \rho \in V_{M}\left(J_{T}\right)$. Problem (3.10) possesses a unique maximally continued solution $u=u(\rho, \varepsilon)$ on some interval $\left[0, t_{\varepsilon}^{+}\right) \subset J_{T}$ in the class $C\left(\left[0, t_{\varepsilon}^{+}\right), h^{2+\alpha}(\bar{\Omega})\right) \cap C^{1}\left(\left[0, t_{\varepsilon}^{+}\right), h^{\alpha}(\bar{\Omega})\right)$. Moreover, $u \geqslant 0$ in $\left[0, t_{\varepsilon}^{+}\right) \times \bar{\Omega}$.

Proof. The first statement follows from Theorem 2.1 in [15]. First assume that $u_{0}>0$ on $\Gamma$. Let $t^{*}:=\sup \left\{0 \leqslant t<t_{\varepsilon}^{+} ; u(t, \cdot)>0\right.$ on $\left.\Gamma\right\}$. Assume $t^{*}<t_{\varepsilon}^{+}$. Then there exists $x \in \Gamma$ such that $u\left(t^{*}, x\right)=0$. Let $D_{t^{*}}:=\left(0, t^{*}\right] \times \Omega$. Taking into account the structure of the mapping $R$ (which is of first order in $u$ and has no zero-order terms) we can conclude from the classical parabolic maximum principle that $u$ achieves its minimal value at the parabolic boundary of $D_{t^{*}}$. Hence, by the strong parabolic maximum principle, $u$ is either constant on $\bar{D}_{t^{*}}$ (and hence non-negative) or $u\left(t^{*}, x\right)$ is a strict minimum in $\left(0, t^{*}\right] \times(\Omega \cup\{x\})$. But the parabolic boundary point Lemma implies that $B(\rho) u\left(t^{*}, x\right)<0$ in that case, contradicting the second equation in (3.10). Thus, $u \geqslant 0$ on $\left[0, t_{\varepsilon}^{+}\right) \times \bar{\Omega}$.

In the case $t^{*}=t_{\varepsilon}^{+}$the maximum principle immediately implies that $u \geqslant 0$.

Suppose now that $u_{0} \geqslant 0$ in $\bar{\Omega}$ and that $u_{0}, \rho_{0}$ are smooth. For $\eta>0$ and suitable $\omega \in \mathbb{R}$ let $u_{\eta}$ be the unique solution of

$$
\left.\begin{array}{rlrl}
\Delta^{2} u_{\eta}+\omega u_{\eta} & =0 & & \text { in } \Omega, \\
u_{\eta} & =\eta & & \text { on } \Gamma, \\
B\left(\rho_{0}\right) u_{\eta} & =-\eta \varepsilon\left(2 u_{0}+\eta+H\left(\rho_{0}\right)\right) & & \text { on } \Gamma .
\end{array}\right\}
$$


Then $\left.u_{\eta}\right|_{\Gamma}=\eta>0$ and $u_{\eta} \rightarrow 0$ in $C^{k}(\bar{\Omega})$ for any $k \in \mathbb{N}$ by elliptic a priori estimates (cf. Section 4, [22]) as $\eta \rightarrow 0$.

For $\gamma>0$ let $\mathcal{O}_{\gamma}$ be a tubular neighborhood of $\Gamma$ with diameter $\gamma$ such that $u_{\eta}>0$ in $\mathcal{O}_{\gamma} \cap \bar{\Omega}$. Let $\psi$ be a smooth cut-off function satisfying $\psi \equiv 1$ in $\overline{\mathcal{O}}_{\gamma / 2} \cap \bar{\Omega}$ and $\psi \equiv 0$ in $\Omega \backslash \overline{\mathcal{O}}_{\gamma}$. Then define $\tilde{u}_{\eta}:=u_{0}+u_{\eta} \cdot \psi$.

By construction this function satisfies $\tilde{u}_{\eta}>0$ on $\Gamma, \tilde{u}_{\eta} \geqslant 0$ in $\bar{\Omega}$ and $B\left(\rho_{0}\right) \tilde{u}_{\eta}=-\varepsilon \tilde{u}_{\eta}\left(\tilde{u}_{\eta}+\right.$ $\left.H\left(\rho_{0}\right)\right)$ on $\Gamma$.

Hence the first part of the proof applies to $\tilde{u}_{\eta}$. The assertion follows now from the continuous dependence of the solution of problem (3.10) on the initial value as stated in Theorem 2.1 in [15] first for $u_{0}, \rho_{0}$ smooth. The general case is obtained by a standard approximation argument.

Lemma 3.5 (Estimation of the Maximum) Given $\rho \in V_{M}\left(J_{T}\right)$, we have

$$
0 \leqslant u(\rho, \varepsilon) \leqslant \max \left\{\sup _{t \in\left[0, t_{\varepsilon}^{+}\right)}|H(\rho(t))|, \max u_{0}\right\} .
$$

Proof. The lower bound follows from the previous Lemma. Due to the maximum principle we have that, given $t \in\left[0, t_{\varepsilon}^{+}\right)$, the function $\left.u\right|_{\bar{\Omega} \times[0, t]}$ achieves its maximal value on the parabolic boundary of $\bar{\Omega} \times[0, t]$. Suppose that this value is achieved on the side, i.e. in a point $\left(t^{*}, x^{*}\right)$ with $t^{*}>0, x^{*} \in \partial \Omega$. Then $Q:=B(\rho) u\left(t^{*}, x^{*}\right) \geqslant 0$. Thus, since $u^{*}:=u\left(t^{*}, x^{*}\right)$ solves the quadratic equation $Y^{2}+H(\rho)\left(t^{*}, x^{*}\right) Y+Q / \varepsilon=0$, we find, letting $h^{*}:=H(\rho)\left(t^{*}, x^{*}\right)$, that $\left(h^{*}\right)^{2} / 4-Q / \varepsilon \geqslant 0$ and

$$
\left|u^{*}\right| \leqslant\left|h^{*}\right| / 2+\sqrt{\left(h^{*}\right)^{2} / 4-Q / \varepsilon} \leqslant\left|h^{*}\right|
$$

and the assertion follows.

Lemma 3.6 Let $u_{0}, \rho_{0}$ be as specified. There is a number $C$ with the following property: If $M>$ $\left\|\rho_{0}\right\|_{h^{4+\beta}(\Gamma)}$ is given, there exist $T^{*}>0, \varepsilon_{0}>0$ such that for all $(T, \varepsilon) \in\left(0, T^{*}\right] \times\left(0, \varepsilon_{0}\right]$ and $\rho \in V=V_{M}\left(J_{T}\right)$ the following holds true:

(i) $t_{\varepsilon}^{+}=T$;

(ii) $u:=u_{\varepsilon} \in B U C\left((0, T), h^{2+\alpha}(\bar{\Omega})\right) \cap B U C^{1}\left((0, T), h^{\alpha}(\bar{\Omega})\right.$;

(iii) $\|u\|_{B U C\left((0, T), h^{2+\alpha}(\bar{\Omega})\right)}+\varepsilon\left\|u_{t}\right\|_{B U C\left((0, T), h^{\alpha}(\bar{\Omega})\right)} \leqslant C$.

Moreover, for each fixed $\varepsilon \in\left(0, \varepsilon_{0}\right], T \in\left(0, T^{*}\right]$ and $\nu \in(0, \alpha)$ the mapping $\rho \mapsto u_{\varepsilon}(\rho)$ : $V_{M}\left(J_{T}\right) \rightarrow C\left(J_{T}, h^{2+v}(\bar{\Omega})\right)$ is continuous.

Proof. In the following, functions $\rho \in V_{M}\left(J_{T}\right)$ are constantly extended to the real line. We first consider the operator

$$
\mathbb{L}:=\mathbb{L}\left(\rho_{0}\right):=\left(\partial_{t}-A\left(\rho_{0}\right)+\lambda, B\left(\rho_{0}\right), \operatorname{tr}_{t=0}\right), \quad \lambda>0 .
$$

If $\lambda>0$ is large enough, by extension and restriction (cf. Lemmas $6.1,6.6$ ) we have that

$$
\begin{aligned}
\sup _{S>0} & \left\|\mathbb{L}^{-1}\right\|_{\mathcal{L}\left(0 \mathbb{G}_{p}([0, S]), \mathbb{E}_{1, p}([0, S])\right)} \\
+\quad \sup _{S>0} & \left\|\mathbb{L}^{-1}\right\|_{\mathcal{L}\left(0 \mathbb{G}([0, S]), \mathbb{E}_{1}([0, S])\right)} \\
& \leqslant L<\infty
\end{aligned}
$$

(and that $\mathbb{L}$ is invertible of course in the corresponding spaces). 
Now let $M>\left\|\rho_{0}\right\|_{h^{4+\beta}(\Gamma)}$ be given. Choose $T^{*}, \varepsilon_{0}>0$ such that the statements of the Lemmata 6.3, 6.4, 6.5 hold true with $\delta=1 /(2 L)$ (in Lemma 6.5). For $\rho \in V_{M}\left(J_{T}\right)\left(T \in\left(0, T^{*}\right]\right)$ let $\rho_{\varepsilon}(t):=\rho(\varepsilon t)\left(\varepsilon \in\left(0, \varepsilon_{0}\right]\right)$ and

$$
\mathbb{L}\left(\rho_{\varepsilon}\right):=\left(\partial_{t}-A\left(\rho_{\varepsilon}\right)+\lambda, B\left(\rho_{\varepsilon}\right), \operatorname{tr}_{t=0}\right) .
$$

Standard results about topological isomorphisms and Lemma 6.5 imply that

$$
\begin{aligned}
\sup _{(T, \varepsilon) \in\left(0, T^{*}\right] \times\left(0, \varepsilon_{0}\right]} & \left\|\mathbb{L}\left(\rho_{\varepsilon}\right)^{-1}\right\|_{\mathcal{L}\left(0 \mathbb{G}_{p}([0, T / \varepsilon]), 0 \mathbb{E}_{1, p}([0, T / \varepsilon])\right.} \\
+\quad \sup _{(T, \varepsilon) \in\left(0, T^{*}\right] \times\left(0, \varepsilon_{0}\right]} & \left\|\mathbb{L}\left(\rho_{\varepsilon}\right)^{-1}\right\|_{\mathcal{L}\left(0 \mathbb{G}([0, T / \varepsilon]), 0_{0} \mathbb{E}_{1}([0, T / \varepsilon])\right.} \\
& \leqslant 4 L .
\end{aligned}
$$

For the remainder of this proof we will denote by the symbol $c$ a generic constant being independent of $(T, \varepsilon) \in\left[0, T^{*}\right] \times\left(0, \varepsilon_{0}\right], M$ and $\rho \in V_{M}\left(J_{T}\right)$.

Let $T \in\left[0, T^{*}\right], \rho \in V_{M}\left(J_{T}\right)$ and let again $\rho_{\varepsilon}(t):=\rho(\varepsilon t)$.

We fix $\psi \in C^{\infty}(\mathbb{R})$ such that $\psi(0)=1, \operatorname{supp}(\psi) \subset \subset(-1 / 2,1 / 2)$ and define $\bar{u}_{0}(t):=$ $u_{0} \psi(t)$. Then the function $w(t)=w(\varepsilon, t):=u(\varepsilon t)-\bar{u}_{0}(t)$ is the unique solution of the scaled system

$$
\mathbb{L}\left(\rho_{\varepsilon}\right) w=\left(F\left(\varepsilon, \rho_{\varepsilon}, w\right), G\left(\varepsilon, \rho_{\varepsilon}, w\right), 0\right) \quad \text { on } \quad J_{\varepsilon},
$$

where $J_{\varepsilon}:=\left[0,\left(t_{\varepsilon}^{+}\right) / \varepsilon\right)$ and

- $F\left(\varepsilon, \rho_{\varepsilon}, w\right):=\varepsilon R\left(\rho_{\varepsilon}, w+\bar{u}_{0}\right)-R_{0}+\lambda w$;

- $G\left(\varepsilon, \rho_{\varepsilon}, w\right):=-\varepsilon\left[w H\left(\rho_{\varepsilon}\right)+2 w \bar{u}_{0}+w^{2}\right]-R_{1}$,

$R_{0}=R_{0}\left(\varepsilon, \rho_{\varepsilon}\right):=\mathbb{L}\left(\rho_{\varepsilon}\right) \bar{u}_{0}, R_{1}=R_{1}\left(\varepsilon, \rho_{\varepsilon}\right):=\varepsilon\left[\bar{u}_{0}^{2}+\bar{u}_{0} H\left(\rho_{\varepsilon}\right)\right]+B\left(\rho_{\varepsilon}\right) \bar{u}_{0}$. Our starting point is the estimate

$$
\|u(t)\|_{C(\bar{\Omega})} \leqslant c, \quad t \in\left[0, t_{\varepsilon}^{+}\right), \quad \text { thus } \quad\|w(t)\|_{C(\bar{\Omega})} \leqslant c, \quad t \in J_{\varepsilon} .
$$

(Lemmata 3.5, 6.4). This implies

$$
\|w\|_{L_{p}\left(\dot{j}_{\varepsilon}, L_{p}(\Omega)\right)} \leqslant c \cdot\left(T^{*}\right)^{1 / p} \cdot \varepsilon^{-1 / p} .
$$

Let $K_{\varepsilon}$ be an arbitrary compact subinterval of $J_{\varepsilon}$, i.e. $K_{\varepsilon}:=\left[0, T_{\varepsilon} / \varepsilon\right], T_{\varepsilon}<t_{\varepsilon}^{+}$. Then $w \in$ ${ }_{0} \mathbb{E}_{1, p}\left(K_{\varepsilon}\right)$ and

$$
\|w\|_{\mathbb{E}_{1, p}\left(K_{\varepsilon}\right)} \leqslant 4 L \cdot\left(\|F\|_{\mathbb{E}_{0, p}\left(K_{\varepsilon}\right)}+\|G\|_{\mathbb{F}_{p}\left(K_{\varepsilon}\right)}\right) .
$$

As $w$ has time trace 0 , we can extend $w=w(\varepsilon)$ from $K_{\varepsilon}$ to the whole of $\mathbb{R}^{+}$according to Lemma 6.6, Remark 6.7. We refrain at this point from giving explicit details about the fact that this extended function defines a right hand side $(F, G)$ in the regularity class $\mathbb{E}_{0, p}\left(\mathbb{R}^{+}\right) \times \mathbb{F}_{p}\left(\mathbb{R}^{+}\right)$. These details will be implicitly contained in the forthcoming considerations. Moreover, our notation will not distinguish between $w$ and its extension.

As $\left.R_{0}\left(\varepsilon, \rho_{\varepsilon}\right)\right|_{[1, \infty)}=\left.R_{1}\left(\varepsilon, \rho_{\varepsilon}\right)\right|_{[1, \infty)}=0$, we find that

$$
\left\|R_{0}\left(\varepsilon, \rho_{\varepsilon}\right)\right\|_{\mathbb{E}_{0, p}\left(\mathbb{R}^{+}\right)}+\left\|R_{1}\left(\varepsilon, \rho_{\varepsilon}\right)\right\|_{\mathbb{F}_{p}\left(\mathbb{R}^{+}\right)} \leqslant c .
$$

Further, using the Lemmata 6.3, 6.4, we first observe that

$$
\begin{aligned}
\left\|H\left(\rho_{\varepsilon}\right) w\right\|_{\mathbb{F}_{p}\left(\mathbb{R}^{+}\right)} & \leqslant c \cdot\left\|H\left(\rho_{\varepsilon}\right) w\right\|_{\mathbb{F}_{p}\left(K_{\varepsilon}\right)} \\
& =c \cdot\left[\left\|H\left(\rho_{\varepsilon}\right) w\right\|_{L_{p}\left(K_{\varepsilon}, W_{p}^{1-1 / p}(\Gamma)\right)}+\left\|H\left(\rho_{\varepsilon}\right) w\right\|_{W_{p}^{(1-1 / p) / 2}\left(K_{\varepsilon}, L_{p}(\Gamma)\right)}\right] \\
& \leqslant c \cdot\left[\|w\|_{\mathbb{E}_{1, p}\left(K_{\varepsilon}\right)}+\left[H\left(\rho_{\varepsilon}\right) w\right]_{\dot{K}_{\varepsilon} ; p,(1-1 / p) / 2 ; L_{p}(\Gamma)}\right] .
\end{aligned}
$$


In the first line we used that $\left.\left(H\left(\rho_{\varepsilon}\right)\right) \cdot w\right)(0)=0$. By the decomposition $H\left(\rho_{\varepsilon}\right)=\left(H\left(\rho_{\varepsilon}\right)-\right.$ $\left.H\left(\rho_{0}\right)\right)+H\left(\rho_{0}\right)$, the Lemmata 6.3, 6.4, 6.2 ii) (with $\left.Y:=C(\Gamma), X:=L_{p}(\Gamma)\right)$ and the observation that $\left[\rho_{\varepsilon}\right]_{\mathbb{R}^{+}, 1, h^{2+\beta}(\Gamma)} \leqslant \varepsilon M$ (cf. Remark 6.8) we find that

$$
\begin{aligned}
{\left[H\left(\rho_{\varepsilon}\right) w\right]_{\dot{K}_{\varepsilon} ; p,(1-1 / p) / 2 ; L_{p}(\Gamma) \leqslant} \leqslant } & \cdot\left(1+M T+M \varepsilon^{(1-1 / p) / 2} T^{(1+1 / p) / 2}\right) \\
& \cdot\|w\|_{\mathbb{F}_{p}\left(K_{\varepsilon}\right)} \\
\leqslant & \cdot\left(1+M T+M \varepsilon^{(1-1 / p) / 2} T^{(1+1 / p) / 2}\right) \\
& \cdot\|w\|_{\mathbb{E}_{1, p}\left(K_{\varepsilon}\right)}
\end{aligned}
$$

(using again the fact that $w(0)=0$ for continuation and restriction in the last estimate). Going further, thanks to (3.16) and $\partial_{i}\left(w^{2}\right)=2 w \partial_{i} w$,

$$
\begin{aligned}
& \left\|w^{2}\right\|_{L_{p}\left(\mathbb{R}^{+}, W_{p}^{1-1 / p}(\Gamma)\right)}^{p} \\
\leqslant & c \cdot\left\|w^{2}\right\|_{L_{p}\left(\mathbb{R}^{+}, W_{p}^{1}(\Omega)\right)}^{N} \\
\leqslant & c \cdot \int_{0}^{\infty}\left(\|w\|_{L_{p}(\Omega)}+\sum_{i=1}^{N}\left\|\partial_{i} w\right\|_{L_{p}(\Omega)}\right)^{p} \\
= & c \cdot \int_{0}^{\infty}\|w\|_{W_{p}^{1}(\Omega)}^{p} \\
\leqslant & c \cdot\|w\|_{\mathbb{E}_{1, p}\left(\mathbb{R}^{+}\right)}^{p},
\end{aligned}
$$

and

$$
\begin{aligned}
\left\|w^{2}\right\|_{W_{p}^{(1-1 / p) / 2}\left(\mathbb{R}^{+}, L_{p}(\Gamma)\right)} & \leqslant c \cdot\|w\|_{L_{\infty}\left(\mathbb{R}^{+}, C(\Gamma)\right)}\|w\|_{W_{p}^{(1-1 / p) / 2}\left(\mathbb{R}^{+}, L_{p}(\Gamma)\right)} \\
& \leqslant c \cdot\|w\|_{W_{p}^{(1-1 / p) / 2}\left(\mathbb{R}^{+}, L_{p}(\Gamma)\right)}
\end{aligned}
$$

thanks again to (3.16) and Lemma 6.2 (i). As

$$
\left\|R\left(w+\bar{u}_{0}, \rho_{\varepsilon}\right)\right\|_{\mathbb{E}_{0, p}\left(\mathbb{R}^{+}\right)}^{p} \leqslant c \cdot\left[1+\int_{0}^{\infty}\|w(t)\|_{W_{p}^{1}(\Omega)}^{p} d t\right] \leqslant c \cdot\left[1+\|w\|_{\mathbb{E}_{1, p}\left(\mathbb{R}^{+}\right)}^{p}\right]
$$

by Lemma 6.3, 6.4 and (3.16), as moreover

$$
\begin{aligned}
\|\lambda w\|_{\mathbb{E}_{0, p}\left(\mathbb{R}^{+}\right)} & \leqslant c \cdot\|\lambda w\|_{\mathbb{E}_{0, p}\left(K_{\varepsilon}\right)} \\
& \leqslant \lambda \cdot c \cdot \varepsilon^{-1 / p}
\end{aligned}
$$

due to (3.16), we have all in all (using (3.18))

$$
\|w\|_{\mathbb{E}_{1, p}\left(K_{\varepsilon}\right)} \leqslant c \cdot \varepsilon \cdot\|w\|_{\mathbb{E}_{1, p}\left(K_{\varepsilon}\right)}+c \cdot \varepsilon^{-1 / p},
$$

hence

$$
\|w\|_{\mathbb{E}_{1, p}\left(K_{\varepsilon}\right)} \leqslant c \cdot \varepsilon^{-1 / p},
$$

provided $\varepsilon_{0}, T^{*}>0$ are small enough.

We want to get back into the Hölder scale. For this we recall the standard embedding results

$$
{ }_{0} \mathbb{E}_{1, p}(J) \hookrightarrow{ }_{0} W_{p}^{\tau}\left(\dot{J}, W_{p}^{s}(\Omega)\right) \hookrightarrow{ }_{0} B U C^{\gamma / 2}\left(\dot{J}, B U C^{\gamma}(\Omega)\right),
$$


where $J \in\{[0, S],[0, \infty)\}, S>0,2 \tau+s \leqslant 2,2 \tau>2 / p+\gamma, s>N / p+\gamma, \gamma>0$ and

$$
{ }_{0} \mathbb{E}_{1, p}(J) \hookrightarrow{ }_{0} B U C\left(\dot{J}, W_{p}^{2-2 / p}\right) \hookrightarrow{ }_{0} B U C\left(\dot{J}, B U C^{1+\gamma}(\Omega)\right),
$$

$\gamma>0$ (such a $\gamma$ exists, since $p>N+2$ ). By extension and restriction (cf. Remark 6.7), we find that the estimates corresponding to the above embeddings do not depend on $S>0$. Summarizing,

$$
\|w\|_{B U C^{\gamma / 2}\left(\dot{K}_{\varepsilon}, B U C^{\gamma}(\Omega)\right)}+\|w\|_{B U C\left(\dot{K}_{\varepsilon}, B U C^{1+\gamma}(\Omega)\right)} \leqslant c \cdot \varepsilon^{-1 / p},
$$

$p>N+2$. We consider now the decomposition $w=a+b$, where

$$
\mathbb{L}\left(\rho_{\varepsilon}\right) a=(\lambda w, 0,0)
$$

and

$$
\mathbb{L}\left(\rho_{\varepsilon}\right) b=(F-\lambda w, G, 0)=\left(\varepsilon R\left(\rho_{\varepsilon}, w+\bar{u}_{0}\right)-R_{0}, G, 0\right) .
$$

Repeating the above arguments in the Hölder scale we obtain the estimate

$$
\|b\|_{\mathbb{E}_{1}\left(K_{\varepsilon}\right)} \leqslant c \cdot \varepsilon^{1-1 / p} \cdot\|w\|_{\mathbb{E}_{1}\left(K_{\varepsilon}\right)}+c .
$$

Here we made use of the estimates

$$
\begin{aligned}
& \left\|z^{2}\right\|_{B U C\left(\dot{\mathbb{R}}^{+}, C^{1+\kappa}(\Gamma)\right)} \leqslant c \cdot\|z\|_{B U C\left(\dot{\mathbb{R}}^{+}, B U C^{\kappa}(\Omega)\right)} \\
& \cdot\|z\|_{B U C\left(\dot{\mathbb{R}}^{+}, B U C^{2+\kappa}(\Omega)\right)} \\
& \left\|z^{2}\right\|_{B U C^{(1+\kappa) / 2}\left(\dot{\mathbb{R}}^{+}, C(\Gamma)\right)} \leqslant c \cdot\|z\|_{B U C\left(\mathbb{R}^{+}, B U C(\Omega)\right)}
\end{aligned}
$$

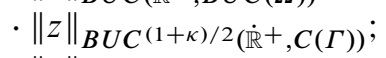

$$
\begin{aligned}
& \left\|z \cdot \partial_{j} z\right\|_{B U C\left(\dot{\mathbb{R}}^{+}, B U C^{\kappa}(\Omega)\right)} \leqslant c \cdot\|z\|_{B U C\left(\dot{\mathbb{R}}^{+}, B U C^{\kappa}(\Omega)\right)} \\
& \text { - }\|z\|_{B U C\left(\dot{\mathbb{R}}^{+}, B U C^{1+\kappa}(\Omega)\right)} \\
& \|r \cdot z\|_{B U C\left(\dot{\mathbb{R}}^{+}, C^{1+\kappa}(\Gamma)\right)} \leqslant c \cdot\|r\|_{B U C\left(\dot{\mathbb{R}}^{+}, C^{1+\kappa}(\Gamma)\right)} \\
& \cdot\|z\|_{B U C\left(\dot{\mathbb{R}}^{+}, B U C^{1+\kappa}(\Omega)\right)} \text {; } \\
& \|r \cdot z\|_{B U C^{(1+\kappa) / 2}\left(\dot{\mathbb{R}}^{+}, C(\Gamma)\right)} \leqslant c \cdot\left(\|r\|_{B U C\left(\dot{\mathbb{R}}^{+}, C(\Gamma)\right)}\|z\|_{B U C^{(1+\kappa) / 2}\left(\dot{\mathbb{R}}^{+}, C(\Gamma)\right)}\right. \\
& \left.+\|r\|_{B U C^{(1+\kappa) / 2}\left(\dot{\mathbb{R}}^{+}, C(\Gamma)\right)}\|z\|_{B U C\left(\dot{\mathbb{R}}^{+}, C(\Gamma)\right)}\right),
\end{aligned}
$$

$\kappa \in(0,1), r, z \in \mathbb{E}_{1}^{\kappa}\left(\mathbb{R}^{+}\right):=B U C\left(\dot{\mathbb{R}}^{+}, h^{2+\kappa}(\bar{\Omega})\right) \cap B U C^{1}\left(\dot{\mathbb{R}}^{+}, h^{\kappa}(\bar{\Omega})\right), j \in\{1, \ldots, N\}$, cf. also Lemma 6.6, Remark 6.7, Theorem 6.1 ii).

Turning now to the function $a$, we observe that this is the unique solution of the evolution equation

$$
\dot{a}-\left(A\left(\rho_{\varepsilon}\right)+\lambda_{1}+\lambda_{2}\right) a=\lambda w, \quad a(0)=0, \quad \lambda_{1}+\lambda_{2}=\lambda,
$$

subject to the time-dependent side condition $B\left(\rho_{\varepsilon}\right) a=0$. Letting

$$
D(t, \varepsilon):=\left\{z \in W_{p}^{2}(\Omega) ; B\left(\rho_{\varepsilon}(t)\right) z=0\right\}
$$

we first observe that by Seeley's classical result (see also Theorem 13.3 [1] for the case of coefficients of finite smoothness)

$$
\left[D(t, \varepsilon), L_{p}(\Omega)\right]_{1 / 2}=W_{p}^{1}(\Omega)
$$

is independent of $t, \varepsilon$. Here, $[\cdot, \cdot]_{\theta}$ denotes the complex interpolation functor. As $\rho_{\varepsilon} \in V_{M}\left(\mathbb{R}^{+}\right)$for $\varepsilon \leqslant 1$, if $\lambda_{1}$ is large enough, we easily verify that the family $Q:=Q(\varepsilon):=\left\{A\left(\rho_{\varepsilon}\right)+\lambda_{1}, B\left(\rho_{\varepsilon}\right)\right\}$ 
satisfies the assumptions (with $\theta=1 / 2$ and $J=[0, \infty)$ ) of Theorem IV 2.6.4 in [2] (thus in particular assumption (2.2.3) in Section IV.2.2 in [2]). Letting $\varepsilon>0$ be small enough $\left(\varepsilon^{3 / 4}<1 / M\right.$ for example, cf. Remark 6.8), we find the constants occurring in the corresponding estimates to be independent of $\varepsilon$ as well as $M$, say, $T \leqslant 1$ and $\rho \in V_{M}\left(J_{T}\right)$. If $U_{\varepsilon}(t, s)$ is the parabolic fundamental solution associated to the family $Q(\varepsilon)$, the unique solution of (3.25) is given by

$$
a(\varepsilon, t):=\lambda \int_{0}^{t} e^{-\lambda_{2}(t-s)} U_{\varepsilon}(t, s) w(s) d s .
$$

If $\lambda_{2}$ is large enough, it follows from Corollary IV. 2.4.2 in [2] and (3.16) that

$$
\|a\|_{B U C\left(\dot{J}_{\varepsilon}, W_{p}^{1}(\Omega)\right)} \leqslant c \cdot \int_{0}^{\infty} \frac{e^{-\bar{\lambda} x}}{\sqrt{x}} d x \leqslant c, \quad \bar{\lambda}>0,
$$

and thus, by Sobolev embedding,

$$
\|a\|_{\mathbb{E}_{0}\left(J_{\varepsilon}\right)} \leqslant c, \quad p>N /(1-\alpha) .
$$

Combining this with (3.24) (as $w=a+b$ ), as $K_{\varepsilon} \subset J_{\varepsilon}$ was arbitrary, we find that

$$
\|w\|_{\mathbb{E}_{0}\left(J_{\varepsilon}\right)} \leqslant c \cdot \varepsilon^{1-1 / p} \cdot\|w\|_{\mathbb{E}_{1}\left(J_{\varepsilon}\right)}+c
$$

and hence, repeating the arguments used to obtain (3.24),

$$
\|w\|_{\mathbb{E}_{1}\left(J_{\varepsilon}\right)} \leqslant c \cdot \varepsilon^{1-1 / p} \cdot\|w\|_{\mathbb{E}_{1}\left(J_{\varepsilon}\right)}+c+\lambda\|w\|_{\mathbb{E}_{0}\left(J_{\varepsilon}\right)} \leqslant c \cdot \varepsilon^{1-1 / p} \cdot\|w\|_{\mathbb{E}_{1}\left(J_{\varepsilon}\right)}+c
$$

i.e.

$$
\|w\|_{\mathbb{E}_{1}\left(J_{\varepsilon}\right)} \leqslant c, \quad \text { thus } \quad\|u\|_{\mathbb{E}_{0}\left(\left(0, t_{\varepsilon}^{+}\right)\right)}+\varepsilon\|\dot{u}\|_{\mathbb{E}_{0}\left(\left(0, t_{\varepsilon}^{+}\right)\right)} \leqslant c,
$$

provided $T^{*}, \varepsilon>0$ are small enough. In particular, $t_{\varepsilon}^{+}=T^{*}, \varepsilon \in\left(0, \varepsilon_{0}\right]$ and (i), (ii), (iii) are proved.

We are left to affirm the continuity of the solution map. First note that classical parabolic theory implies that, given $\varepsilon>0$ we have an estimate of the form

$$
\left\|u_{\varepsilon}(\rho)\right\|_{C^{2+\alpha,(2+\alpha) / 2([0, T] \times \bar{\Omega})} \leqslant} \leqslant C_{\varepsilon} \quad\left(T \leqslant T^{*}\right),
$$

uniformly in $\rho \in V_{M}\left(J_{T}\right)$ (but not uniformly in $\varepsilon$ of course). Let $\left(\rho_{n}\right) \subset V_{M}\left(J_{T}\right)\left(T \leqslant T^{*}\right)$ converge to some $\sigma \in V_{M}\left(J_{T}\right)$. Then (3.27) and compact embedding imply that there is a subsequence $\left(\rho_{n_{k}}\right)$ such that $u_{\varepsilon}\left(\rho_{n_{k}}\right) \rightarrow v \in C^{2+v,(2+v) / 2}([0, T] \times \bar{\Omega})$, where $v<\alpha$. As $v$ clearly solves the limit problem associated to $\sigma$, and as the solution of this problem is unique, we infer that actually $u_{\varepsilon}\left(\rho_{n}\right) \rightarrow v=u_{\varepsilon}(\sigma)$ in $C\left([0, T], h^{2+v}(\bar{\Omega})\right)$. This finally proves the theorem.

\subsection{The fixed point argument}

In this section let $\mathbb{Y}_{\gamma}\left(J_{T}\right):=C\left(J_{T}, h^{4+\gamma}(\Gamma)\right) \cap C^{1}\left(J_{T}, h^{2+\gamma}(\Gamma)\right)$ and $\mathbb{Z}_{\gamma}\left(J_{T}\right):=$ $C\left(J_{T}, h^{2+\gamma}(\Gamma)\right), \gamma \in[\beta, \alpha]$. The uniformly elliptic operator $P\left(\rho_{0}\right)$ can be assumed to fulfill

$$
\left(\partial_{t}-L_{\rho_{0}} P\left(\rho_{0}\right), \operatorname{tr}_{t=0}\right) \in \mathcal{L}_{\text {is }}\left(\mathbb{Y}_{\gamma}\left(J_{T}\right), \mathbb{Z}_{\gamma}\left(J_{T}\right) \times h^{2+\gamma}(\Gamma)\right),
$$


where $T \in(0, \infty)$, and

$$
\sup _{0<T \leqslant 1}\left\|\left(\partial_{t}-L_{\rho_{0}} P\left(\rho_{0}\right), \operatorname{tr}_{t=0}\right)^{-1}\right\|_{\mathcal{L}\left(\mathbb{Z}_{\gamma}\left(J_{T}\right) \times h^{2+\gamma}(\Gamma), \mathbb{Y}_{\gamma}\left(J_{T}\right)\right)} \leqslant L=L(\gamma)<\infty
$$

(cf. Theorem III 3.4.1 in [2]). Thus from the Lemmas 6.3, 6.4 we get

Lemma 3.7 Given $M>\left\|\rho_{0}\right\|_{h^{4+\beta}(\Gamma)}$, there is a $T^{*}>0$ such that for all $T \in\left(0, T^{*}\right]$ and $\rho \in V_{M}\left(J_{T}\right)$ we have

- $\left\|L_{\rho} P(\rho)-L_{\rho_{0}} P\left(\rho_{0}\right)\right\|_{\mathcal{L}\left(\mathbb{Y}_{\gamma}\left(J_{T}\right), \mathbb{Z}_{\gamma}\left(J_{T}\right)\right)} \leqslant 1 /(2 L)$, thus

- $\left\|\left(\partial_{t}-L_{\rho} P(\rho), t r_{t=0}\right)^{-1}\right\|_{\mathcal{L}\left(\mathbb{Z}_{\gamma}\left(J_{T}\right) \times h^{2+\gamma}(\Gamma), \mathbb{Y}_{\gamma}\left(J_{T}\right)\right)} \leqslant 2 L$.

Here $L_{\rho_{0}}$, $L_{\rho}$ denote the functions introduced in (3.2).

Now we can prove the following result:

Theorem 3.8 There are $T^{*}, \varepsilon_{0}>0$ such that the following holds true:

- For any $\varepsilon \in\left(0, \varepsilon_{0}\right]$ the transformed system (3.3) possesses a unique solution $\left(u_{\varepsilon}, \rho_{\varepsilon}\right) \in \mathbb{E}_{1}\left(J_{T^{*}}\right) \times$ $\mathbb{Y}_{\alpha}\left(J_{T^{*}}\right)$.

Proof. Choose $\tilde{L}>0$ such that $\sup _{\zeta \in B_{a}}\left(\left\|L_{\zeta}\right\|_{h^{2, \gamma}(\Gamma)}+\left\|L_{\zeta} Q(\zeta)\right\|_{h^{2, \gamma}(\Gamma)}\right) \leqslant \tilde{L}, \gamma \in\{\beta, \alpha\}$ (Lemma 6.3). Choose $L>0$ according to the above considerations. Let $C$ be the constant from Lemma 3.6. Let $M>4 L\left(\tilde{L}(1+C)+\left\|\rho_{0}\right\|_{h^{4+\beta}(\Gamma)}\right), T>0, \rho \in V_{M}\left(J_{T}\right)$. Then let $\sigma$ solve

$$
\partial_{t} \sigma-L_{\rho} P(\rho) \sigma=L_{\rho}\left(Q(\rho)+\left.u_{\varepsilon}(\rho)\right|_{\Gamma}\right), \quad \sigma(0)=\rho_{0}
$$

We have (assuming w.l.o.g. that $C$ is an upper bound also for $\left.\left\|\left.u_{\varepsilon}(\rho)\right|_{\Gamma}\right\|_{C\left(J_{T}, h^{2+\gamma}(\Gamma)\right.}, \gamma \in\{\beta, \alpha\}\right)$

$$
\begin{aligned}
\|\sigma\|_{\mathbb{Y}_{\beta}}\left(J_{T}\right) \leqslant & \left\|\left(\partial_{t}-L_{\rho} P(\rho), \operatorname{tr}_{t=0}\right)^{-1}\right\|_{\mathcal{L}\left(\mathbb{Z}_{\beta}\left(J_{T}\right), \mathbb{Y}_{\beta}\left(J_{T}\right)\right)} \\
& \cdot\left\|\left(L_{\rho}\left(Q(\rho)+u_{\varepsilon}(\rho)\right), \rho_{0}\right)\right\|_{\mathbb{Z}_{\beta}\left(J_{T}\right) \times h^{4+\beta}(\Gamma)} \\
\leqslant & 2 L\left(\tilde{L}(1+C)+\left\|\rho_{0}\right\|_{h^{4+\beta}(\Gamma)}\right) \\
< & M,
\end{aligned}
$$

provided $T, \varepsilon$ are small enough and thus

$$
\rho \mapsto \sigma: V_{M}\left(J_{T}\right) \rightarrow V_{M}\left(J_{T}\right)
$$

(thanks to Lemma 6.4 we can assume $\sigma$ to take its values in Ad). Using the continuity of $\rho \mapsto$ $u_{\varepsilon}(\rho)$, the mapping (3.28) is easily seen to be continuous. Moreover, as $\rho_{0} \in h^{4+\alpha}(\Gamma)$, one easily concludes that in fact

$$
\sup _{\rho \in V_{M}\left(J_{T}\right)}\|\sigma(\rho)\|_{C\left(J_{T}, h^{4+\alpha}(\Gamma)\right)}<\infty .
$$

Thus, by the compactness of the embedding $h^{4+\alpha}(\Gamma)$ into $h^{4+\beta}(\Gamma)$, by the Schauder fixed point theorem there is a fixed point $\rho_{\varepsilon}$ inside $V_{M}\left(J_{T}\right)$. The uniqueness follows from the uniqueness result obtained in [12].

\subsection{The convergence of the solution}

In this section we denote by $\left(u_{\varepsilon}, \rho_{\varepsilon}\right):\left[0, T^{*}\right] \rightarrow \mathbb{R}^{\bar{\Omega}} \times \mathbb{R}^{\Gamma}$ the solution of problem (3.3). By construction we have

$$
\bigcup_{0<\varepsilon<\varepsilon_{0}} \rho_{\varepsilon} \subset V_{M}\left(J_{T^{*}}\right), \quad \sup _{0<\varepsilon<\varepsilon_{0}}\left\|u_{\varepsilon}\right\|_{C\left(J_{\left.T^{*}, h^{2+\alpha}(\bar{\Omega})\right)}<\infty .\right.}
$$


Further, also by construction, we have

$$
\sup _{\varepsilon \in\left(0, \varepsilon_{0}\right]}\left\|\rho_{\varepsilon}\right\|_{\mathbb{Y}_{\alpha}\left(J_{T^{*}}\right)} \leqslant s_{0}<\infty
$$

From the first relation in (3.29), (3.30) and a standard interpolation argument for (little) Hölder spaces we conclude that

$$
\begin{aligned}
\left\|\rho_{\varepsilon}(t)-\rho_{\varepsilon}(s)\right\|_{h^{4+\beta}(\Gamma)} & \leqslant c(\alpha, \beta) \cdot\left\|\rho_{\varepsilon}(t)-\rho_{\varepsilon}(s)\right\|_{h^{2+\beta}(\Gamma)}^{\frac{\alpha-\beta}{2+\alpha-\beta}}\left\|\rho_{\varepsilon}(t)-\rho_{\varepsilon}(s)\right\|_{h^{4+\alpha}(\Gamma)}^{\frac{2}{2+\alpha-\beta}} \\
& \leqslant c(\alpha, \beta) \cdot M \cdot\left(2 s_{0}\right)^{\frac{2}{2+\alpha-\beta}} \cdot|t-s|^{\frac{\alpha-\beta}{2+\alpha-\beta}} .
\end{aligned}
$$

Hence, the family $\left\{\rho_{\varepsilon} ; 0<\varepsilon<\varepsilon_{0}\right\}$ (considered as a subset of $C\left(J_{T^{*}}, h^{4+\beta}(\Gamma)\right)$ ) is uniformly equicontinuous. Since the embedding $h^{b}(\Gamma) \hookrightarrow h^{a}(\Gamma)(a<b)$ is compact, we conclude from (3.30) and the Arzelà-Ascoli Theorem that there is a function $\rho \in C\left(J_{T^{*}}, h^{4+\beta}(\Gamma)\right)$ such that

$$
\rho_{\varepsilon} \rightarrow \rho \quad \text { in } C\left(J_{T^{*}}, h^{4+\beta}(\Gamma)\right) .
$$

REMARK 3.9 The convergence in (3.31) has of course to be understood in the sense of possibly passing to a subnet. As our final limit at the end of the day will be the unique solution of the equation (2.5), this is an unnecessary constraint. Therefore we disclaim of this precision at this point.

Turning to the functions $u_{\varepsilon}$ we will present a different approach now than presented in [11]: Instead of estimating the modulus of continuity of the $u_{\varepsilon}$, we prove the convergence result in a direct way: Recall that $\Gamma_{\rho_{\varepsilon}}(t)=\theta_{\rho_{\varepsilon}(t)}[\Gamma], \Omega_{\rho_{\varepsilon}}(t)=\theta_{\rho_{\varepsilon}(t)}[\Omega]$ and that $V_{n}(\varepsilon):=V_{n}\left(\Gamma_{\rho_{\varepsilon}}\right)=\dot{\rho}_{\varepsilon} /\left|\nabla \varphi_{\rho_{\varepsilon}}\right|$. Therefore, by (3.29), we find constants $\alpha_{0}, \alpha_{1}>0$ such that

$$
\left|V_{n}(\varepsilon)(t)\right| \leqslant \alpha_{1}, \quad 0<\alpha_{0} \leqslant \operatorname{vol}\left(\Omega_{\rho_{\varepsilon}}(t)\right) \leqslant \alpha_{1}, \quad(t, \varepsilon) \in\left[0, T^{*}\right] \times\left(0, \varepsilon_{0}\right] .
$$

Moreover, letting $v_{\varepsilon}:=u_{\varepsilon} \circ \theta_{\rho_{\varepsilon}}^{-1}$ (i.e. $v_{\varepsilon}$ solves the untransformed model (2.2)), we have that by the conservation of mass and (I2)

$$
\int_{\Omega_{\rho_{\varepsilon}(t)}} v_{\varepsilon}(t)=\int_{\Omega_{\rho_{0}}} v_{0}=: M_{0}, \quad(t, \varepsilon) \in\left[0, T^{*}\right] \times\left(0, \varepsilon_{0}\right]
$$

Compactness arguments show that the family of surfaces $\left\{\Gamma_{\rho_{\varepsilon}(t)} ; \varepsilon \in\left(0, \varepsilon_{0}\right] ; t \in\left[0, T^{*}\right]\right\} \cup$ $\left\{\Gamma_{\rho(t)} ; t \in\left[0, T^{*}\right]\right\}$ satisfies the uniform cone property needed for Theorem 1 (Section 3) in [3]. Hence, by this Theorem, there holds the following uniform Poincaré-inequality:

$$
\left\|v_{\varepsilon}(t)-\frac{M_{0}}{\operatorname{vol}\left(\Omega_{\rho_{\varepsilon}(t)}\right)}\right\|_{L_{p}\left(\Omega_{\left.\rho_{\varepsilon}(t)\right)}\right.} \leqslant C_{P} \cdot\left\|\nabla v_{\varepsilon}(t)\right\|_{L_{p}\left(\Omega_{\rho_{\varepsilon}}(t)\right)}, \quad p \in[1, \infty),
$$

$C_{P}$ being independent of $(t, \varepsilon) \in\left[0, T^{*}\right] \times\left(0, \varepsilon_{0}\right]$. Therefore, using Transport and Divergence Theorem, the uniform Poincaré estimate and (3.29) (and writing $V_{n}, v$ instead of $V_{n}(\varepsilon), v_{\varepsilon}$ for 
simplicity), we find

$$
\begin{aligned}
& \frac{d}{d t}\left\|v-\frac{M_{0}}{\operatorname{vol}\left(\Omega_{\rho_{\varepsilon}(t)}\right)}\right\|_{L_{2}\left(\Omega_{\left.\rho_{\varepsilon}(t)\right)}\right.}^{2}=2 \int_{\Omega_{\rho_{\varepsilon}(t)}}\left(v-\frac{M_{0}}{\operatorname{vol}\left(\Omega_{\rho_{\varepsilon}(t)}\right)}\right)\left(\dot{v}+\frac{M_{0} \int_{\Gamma_{\rho_{\varepsilon}(t)}} V_{n}}{\operatorname{vol}\left(\Omega_{\rho_{\varepsilon}(t)}\right)^{2}}\right) \\
& +\int_{\Gamma_{\rho_{\varepsilon}(t)}} V_{n}\left(v-\frac{M_{0}}{\operatorname{vol}\left(\Omega_{\rho_{\varepsilon}(t)}\right)}\right)^{2} \\
& =2 / \varepsilon \int_{\Gamma_{\rho_{\varepsilon}(t)}} \partial_{n} v\left(v-\frac{M_{0}}{\operatorname{vol}\left(\Omega_{\rho_{\varepsilon}(t)}\right)}\right)-2 / \varepsilon \int_{\Gamma_{\rho_{\varepsilon}(t)}}|\nabla v|^{2} \\
& +2 \int_{\Omega_{\rho_{\varepsilon}(t)}}\left(v-\frac{M_{0}}{\operatorname{vol}\left(\Omega_{\rho_{\varepsilon}(t)}\right)}\right)\left(\frac{M_{0} \int_{\Gamma_{\varepsilon}} V_{n}}{\operatorname{vol}\left(\Omega_{\rho_{\varepsilon}(t)}\right)^{2}}\right) \\
& +\int_{\Gamma_{\rho_{\varepsilon}(t)}} V_{n}\left(v-\frac{M_{0}}{\operatorname{vol}\left(\Omega_{\rho_{\varepsilon}(t)}\right)}\right)^{2} \\
& =2 \int_{\Gamma_{\rho_{\varepsilon}(t)}}\left(v^{2}+v H\left(\Gamma_{\rho_{\varepsilon}(t)}\right)\right)\left(v-\frac{M_{0}}{\operatorname{vol}\left(\Omega_{\rho_{\varepsilon}(t)}\right)}\right) \\
& -2 / \varepsilon \int_{\Gamma_{\rho_{\varepsilon}(t)}}|\nabla v|^{2} \\
& +2 \int_{\Omega_{\rho_{\varepsilon}(t)}}\left(v-\frac{M_{0}}{\operatorname{vol}\left(\Omega_{\rho_{\varepsilon}(t)}\right)}\right)\left(\frac{M_{0} \int_{\Gamma_{\varepsilon}} V_{n}}{\operatorname{vol}\left(\Omega_{\rho_{\varepsilon}(t)}\right)^{2}}\right) \\
& +\int_{\Gamma_{\rho_{\varepsilon}(t)}} V_{n}\left(v-\frac{M_{0}}{\operatorname{vol}\left(\Omega_{\rho_{\varepsilon}(t)}\right)}\right)^{2} \\
& \leqslant-2 C_{P}^{-2} / \varepsilon \cdot\left\|v(t)-\frac{M_{0}}{\operatorname{vol}\left(\Omega_{\rho_{\varepsilon}(t)}\right)}\right\|_{L_{2}\left(\Omega_{\rho_{\varepsilon}(t)}\right)}^{2}+c,
\end{aligned}
$$

and thus

$$
\frac{d}{d t} \ln \left\|v-\frac{M_{0}}{\operatorname{vol}\left(\Omega_{\rho_{\varepsilon}(t)}\right)}\right\|_{L_{2}\left(\Omega_{\rho_{\varepsilon}}(t)\right)}^{2} \leqslant-2 C_{P}^{-2} / \varepsilon+c /\left\|v-\frac{M_{0}}{\operatorname{vol}\left(\Omega_{\rho_{\varepsilon}(t)}\right)}\right\|_{L_{2}\left(\Omega_{\rho_{\varepsilon}}(t)\right)}^{2} .
$$

Thus, if $\left\|v-\frac{M_{0}}{\operatorname{vol}\left(\Omega_{\rho_{\varepsilon}(t)}\right)}\right\|_{L_{2}\left(\Omega_{\rho_{\varepsilon}}(t)\right)}>\varepsilon^{1 / 4}$, then

$$
\frac{d}{d t} \ln \left\|v-\frac{M_{0}}{\operatorname{vol}\left(\Omega_{\rho_{\varepsilon}(t)}\right)}\right\|_{L_{2}\left(\Omega_{\rho_{\varepsilon}}(t)\right)}^{2} \leqslant-2 C_{P}^{-2} / \varepsilon+c / \sqrt{\varepsilon},
$$

hence

$$
\left\|v-\frac{M_{0}}{\operatorname{vol}\left(\Omega_{\rho_{\varepsilon}(t)}\right)}\right\|_{L_{2}\left(\Omega_{\rho_{\varepsilon}}(t)\right)}^{2} \leqslant\left\|v_{0}-\frac{M_{0}}{\operatorname{vol}\left(\Omega_{\rho_{0}}\right)}\right\|_{L_{2}\left(\Omega_{\rho_{0}}\right)}^{2} e^{-2 C_{P}^{-2} t / \varepsilon+c t / \sqrt{\varepsilon}},
$$

and, finally summarizing and after possibly shrinking the size of $\varepsilon_{0}$,

$$
\left\|v-\frac{M_{0}}{\operatorname{vol}\left(\Omega_{\rho_{\varepsilon}(t)}\right)}\right\|_{L_{2}\left(\Omega_{\rho_{\varepsilon}}(t)\right)}^{2} \leqslant \max \left\{c \cdot e^{-\tilde{c} t / \varepsilon}, \sqrt{\varepsilon}\right\} .
$$


Combining this with (3.29), we obtain for the function $u=v \circ \theta_{\rho_{\varepsilon}}$ by repeated interpolation and Sobolev embedding arguments that

$$
\left\|u-\frac{M_{0}}{\operatorname{vol}\left(\Omega_{\rho_{\varepsilon}(t)}\right)}\right\|_{h^{2+\beta}(\bar{\Omega})} \rightarrow 0
$$

as $\varepsilon \rightarrow 0$, uniformly on compact intervals $[\delta, T]$, where $0<\delta<T \leqslant T^{*}$. The assertion of Theorem 3.1 follows now from Theorem 3.8, (3.29) and the observation

$$
\frac{M_{0}}{\operatorname{vol}\left(\Omega_{\rho_{\varepsilon}(t)}\right)} \rightarrow \frac{M_{0}}{\operatorname{vol}\left(\Omega_{\rho(t)}\right)}
$$

as $\varepsilon \rightarrow 0$, uniformly on $\left[0, T^{*}\right]$ (this follows straightforwardly from (3.31).

Note that from the variation of constants formula we infer that $\rho$ is a mild solution of $\dot{\tau}=$ $L_{\tau}\left(H(\tau)+\frac{M_{0}}{\operatorname{vol}\left(\Omega_{\tau}\right)}\right)\left(\tau(0)=\rho_{0}\right)$, thus, by its regularity, a classical one. The uniqueness of the solution of this equation is proven in [26].

\section{The Stefan problem with Gibbs-Thomson correction and kinetic undercooling}

In this section we sketch briefly, how our previous results can be used to justify the quasistationary approximation of the one phase Stefan problem with Gibbs-Thomson correction and kinetic undercooling. Recall the problem from Section 2:

$$
\left.\begin{array}{rlrl}
\varepsilon \partial_{t} v-\Delta v & =0 & & \text { in } \Omega(t), \\
\partial_{n} v & =-v-H & & \text { on } \Gamma(t), \\
V_{n} & =H+v & & \text { on } \Gamma(t) .
\end{array}\right\}
$$

Transformation to a reference domain $\Omega$ of (4.1) yield (letting $\Gamma:=\partial \Omega$ ):

$$
\left.\begin{array}{rlrl}
\varepsilon \partial_{t} u-A(\rho) u & =\varepsilon R(\rho, u) & & \text { in } \Omega \times(0, T], \\
B(\rho) u & =-u-H(\rho) & & \text { on } \Gamma \times[0, T], \\
\partial_{t} \rho & =L_{\rho} H(\rho)+L_{\rho} u & & \text { on } \Gamma \times(0, T], \\
u(0) & =u_{0} & & \text { in } \Omega, \\
\rho(0) & =\rho_{0} & & \text { on } \Gamma .
\end{array}\right\}
$$

Theorem 4.1 Let $0<\beta<\alpha<1$ and $\left(u_{0}, \rho_{0}\right) \in h^{2+\alpha}(\bar{\Omega}) \times h^{4+\alpha}(\Gamma) \cap$ Ad be subject to the compatibility condition

$$
B\left(\rho_{0}\right) u_{0}+u_{0}=-H\left(\rho_{0}\right) \quad \text { on } \Gamma .
$$

Then there exist $T^{*}, \varepsilon_{0}>0$ with the following properties:

- For each $0<\varepsilon<\varepsilon_{0}$ system (4.2) possesses a unique classical solution $\left(u_{\varepsilon}, \rho_{\varepsilon}\right) \in \mathbb{E}_{1}\left(J_{T^{*}}\right) \times$ $\mathbb{Y}_{\alpha}\left(J_{T^{*}}\right)$.

- $\left(u_{\varepsilon}, \rho_{\varepsilon}\right) \stackrel{\varepsilon \rightarrow 0}{\longrightarrow}(y, \sigma)$, where $(y, \sigma) \in C\left([0, T], h^{3+\alpha}(\bar{\Omega})\right) \times \mathbb{Y}_{\alpha}\left(J_{T^{*}}\right)$ is the unique solution of (4.2) with $\varepsilon=0$, i.e. for all $t \in\left[0, T^{*}\right]$ we have

$$
\left.\begin{array}{rlrl}
A(\sigma(t)) y(t) & =0 & & \text { in } \Omega, \\
B(\sigma(t)) y(t) & =-y(t)-H(\sigma(t)) & & \text { on } \Gamma, \\
\sigma(0) & =\rho_{0} & & \text { on } \Gamma,
\end{array}\right\}
$$


and, additionally, for all $t \in\left(0, T^{*}\right]$ it holds

$$
\sigma_{t}(t)=L_{\sigma(t)}(H(\sigma(t))+y(t)) \quad \text { on } \Gamma .
$$

The convergence takes place in $C\left(\left[\delta, T^{*}\right], h^{2+\beta}(\bar{\Omega})\right) \times C\left(\left[0, T^{*}\right], h^{4+\beta}(\Gamma)\right)$ for each $\delta \in\left(0, T^{*}\right)$.

Large parts of the proof are similar to arguments used for the osmosis problem. We sketch the most important differences:

Proof. - The maximum estimate: Let $\rho \in V_{M}\left(J_{T}\right)$. As in a maximum (minimum) point at $(x, t) \in$ $\Gamma \times\left(0, t_{\varepsilon}^{+}\right)$we have $B(\rho) u \geqslant 0(B(\rho) u \leqslant 0)$ we immediately get

$$
\left|u_{\varepsilon}(t, x)\right| \leqslant \max \left\{\left|u_{0}\right|, \max _{(t, x) \in[0, T] \times \Gamma}|H(\rho)(t, x)|\right\} .
$$

- The stronger a priori estimates: Starting with this maximum estimate, the first statement of Theorem 4.1 can be proved along the lines of Sections 3.2, 3.3. In particular, one obtains the crucial a priori estimate

$$
\left\|u_{\varepsilon}(\rho)\right\|_{C\left(J_{T}, h^{2, \alpha}(\bar{\Omega})\right)}+\varepsilon\left\|\dot{u}_{\varepsilon}(\rho)\right\|_{\mathbb{E}_{0}\left(J_{T}\right)} \leqslant C .
$$

- The convergence of the solution: To finally establish the convergence result we give an argument related to the isoperimetric inequality for the Robin-Laplacian.

Denoting by $\left(u_{\varepsilon}, \rho_{\varepsilon}\right) \subset \mathbb{E}_{1}\left(J_{T^{*}}\right) \times \mathbb{Y}_{\alpha}\left(J_{T^{*}}\right)$ the solutions of (4.2), we have by construction that

$$
\rho_{\varepsilon} \rightarrow \sigma \in V_{M}\left(J_{T^{*}}\right) \quad \text { in } C\left(\left[0, T^{*}\right], h^{4+\beta}(\Gamma)\right)
$$

(in the sense of Remark 3.9). Let $y=y(\varepsilon, t)$ solve the elliptic problem

$$
\left.\begin{array}{rlrl}
A\left(\rho_{\varepsilon}\right) y & =0 & & \text { in } \Omega, \\
B\left(\rho_{\varepsilon}\right) y+y & =-H\left(\rho_{\varepsilon}\right) & & \text { on } \Gamma,
\end{array}\right\}
$$

i.e. $y=-T\left(\rho_{\varepsilon}\right) H\left(\rho_{\varepsilon}\right)$, where $T(\tau) g$ denotes the unique solution of the linear elliptic problem

$$
\begin{aligned}
& A(\tau) y=0 \quad \text { in } \Omega, \text {, } \\
& B(\tau) y+y=g \quad \text { on } \Gamma .\}
\end{aligned}
$$

Observe that $T \in C^{\infty}\left(h^{2+v}(\Gamma) \cap \operatorname{Ad}, \mathcal{L}\left(h^{v}(\Gamma), h^{1+v}(\bar{\Omega})\right), v \in(0,2)\right.$. Thus,

$$
-\partial_{t} y=D T\left(\rho_{\varepsilon}\right)\left(\rho_{\varepsilon}\right)_{t} H\left(\rho_{\varepsilon}\right)+T\left(\rho_{\varepsilon}\right) D H\left(\rho_{\varepsilon}\right)\left(\rho_{\varepsilon}\right)_{t} \in C\left(\left[0, T^{*}\right], h^{\alpha}(\bar{\Omega})\right)
$$

and $\left\|\partial_{t} y(\varepsilon, t)\right\|_{h^{\alpha}(\bar{\Omega})} \leqslant c$ uniformly in $(t, \varepsilon) \in\left[0, T^{*}\right] \times\left(0, \varepsilon_{0}\right]$. Letting $z:=z_{\varepsilon}:=u_{\varepsilon}-y$ we have

$$
\begin{aligned}
\partial_{t} z-1 / \varepsilon A\left(\rho_{\varepsilon}\right) z & =R\left(u_{\varepsilon}, \rho_{\varepsilon}\right)-\partial_{t} y & & \text { in } \Omega \times\left(0, T^{*}\right] \\
B\left(\rho_{\varepsilon}\right) z+z & =0 & & \text { on } \Gamma \times\left[0, T^{*}\right], \\
z(0) & =u_{\varepsilon}(0)-y(0) & & \text { in } \Omega
\end{aligned}
$$

Let $\kappa:=\kappa(\varepsilon, t):=\left|\operatorname{det}\left(D \theta_{\rho_{\varepsilon}(t)}\right)\right|$,

$$
\begin{aligned}
0<\kappa_{0} & :=\inf \left\{\kappa(\varepsilon, t) ; \varepsilon \in\left(0, \varepsilon_{0}\right] ; t \in\left[0, T^{*}\right]\right\} \\
& \leqslant \sup \left\{\kappa(\varepsilon, t) ; \varepsilon \in\left(0, \varepsilon_{0}\right] ; t \in\left[0, T^{*}\right]\right\}=: \kappa_{1}<\infty
\end{aligned}
$$


and $D(\varepsilon, t):=\left\{h \in W_{2}^{2}(\Omega, \mathbb{C}) ; B\left(\rho_{\varepsilon}(t)\right) h+h=0\right.$ on $\left.\Gamma\right\}$ as well as $A_{\omega}:=A_{\omega}(\varepsilon, t):=$ $A\left(\rho_{\varepsilon}(t)\right)+\omega, \tilde{A}:=\tilde{A}(\varepsilon, t):=\kappa A\left(\rho_{\varepsilon}(t)\right), \tilde{A}_{\omega}:=\tilde{A}(\varepsilon, t):=\kappa A_{\omega}(\omega>0$ to be determined later). The functional

$$
\ell(\varepsilon, t): D(\varepsilon, t) \rightarrow \mathbb{C}, \quad f \mapsto \int_{\Omega}-\kappa \tilde{A} f \cdot \bar{f}
$$

satisfies (letting $f_{\varepsilon}:=f_{\rho_{\varepsilon}(t)}:=f \circ \theta_{\rho_{\varepsilon}(t)}^{-1}$ )

$$
\begin{aligned}
\ell(\varepsilon, t) f & =\int_{\Omega_{\varepsilon}(t)}\left|\nabla f_{\varepsilon}\right|^{2}+\int_{\Gamma} \kappa|f|^{2}-\int_{\Omega} \kappa \omega|f|^{2} . \\
& =: \mathcal{K} f-\mathcal{K}_{\omega} f .
\end{aligned}
$$

Obviously, $\mathcal{K}(\varepsilon, t) f \geqslant 0$ for any $f \in D(\varepsilon, t)$. Let $\rho_{\varepsilon=0}:=\sigma$ and

$$
m:=\bigcup_{(\varepsilon, t) \in\left[0, \varepsilon_{0}\right] \times\left[0, T^{*}\right]}\{(\varepsilon, t)\} \times\left[D(\varepsilon, t) \cap \partial \mathbb{B}(0,1)_{L_{2}(\Omega)}\right] \subset \mathbb{R}^{2} \times W_{2}^{2}(\Omega, \mathbb{C}) .
$$

We shall see that $\mathcal{K}(\varepsilon, t) f$ defined on $\boldsymbol{M}$ is bounded away from 0 . Indeed, suppose that there is a (possibly constant) minimizing sequence $\left(\varepsilon_{n}, t_{n}, f_{n}\right) \subset m$. By construction, the Jacobi matrices $\left\{D \theta_{\rho_{\varepsilon}(t)} ;(\varepsilon, t) \in\left[0, \varepsilon_{0}\right] \times\left[0, T^{*}\right]\right\}$ are 'uniformly invertible'. Hence $\left\|\nabla f_{n}\right\|_{L_{2}(\Omega)} \rightarrow 0$ and compactness arguments imply that (after possibly passing to a subsequence) $\left(\varepsilon_{n}, t_{n}, f_{n}\right) \rightarrow$ $(\tilde{\varepsilon}, \tilde{t}, f) \in\left[0, \varepsilon_{0}\right] \times\left[0, T^{*}\right] \times L_{2}(\Omega)$. Moreover, the Poincaré inequality implies that $f=$ $\frac{1}{|\Omega|} \int_{\Omega} f=: \tilde{\omega}$. Taking the trace on $\partial \Omega$, we get $\left\|\tilde{\omega}-f_{n}\right\|_{W_{2}^{1 / 2}(\Gamma)} \rightarrow 0$. Since $\left\|f_{n}\right\|_{L_{2}(\Gamma)} \rightarrow 0$, we necessarily have $\tilde{\omega}=0$, which contradicts the fact that $\left\|f_{n}\right\|_{L_{2}(\Omega)}=1$ for all $n$. Hence,

$$
\inf \left\{\mathcal{K}(\varepsilon, t) f ; \quad f \in D(\varepsilon, t) ; \quad\|f\|_{L_{2}(\Omega)}=1 ; \quad(\varepsilon, t) \in\left(0, \varepsilon_{0}\right] \times\left(0, T^{*}\right]\right\} \geqslant \omega_{0}>0 .
$$

Observe that the numerical ranges of the operators $\tilde{A}$ and $\tilde{A}_{\omega}$ are the sets $S_{\tilde{A}}:=\{-\mathcal{K}(\varepsilon, t) f ; f \in$ $\left.D(\varepsilon, t) ;\|f\|_{L_{2}(\Omega)}=1\right\}$ and $S_{\tilde{A}_{\omega}}:=\left\{-l(\varepsilon, t) f ; f \in D(\varepsilon, t) ;\|f\|_{L_{2}(\Omega)}=1\right\}$. Hence, choosing $\omega>0$ small enough (and using Hölder's inequality to obtain the second $\geqslant$ ),

$$
\begin{aligned}
\|(\lambda-C) f\|_{L_{2}(\Omega)} & \geqslant 1 / \kappa_{1}\|(\kappa \lambda-\tilde{C}) f\|_{L_{2}(\Omega)} \\
& \geqslant 1 /\left.\kappa_{1}\left|\int_{\Omega} \kappa \lambda\right| f\right|^{2}-\int_{\Omega} \bar{f} \tilde{C} f \mid \\
& \geqslant 1 / \kappa_{1} \operatorname{dist}\left(\int_{\Omega} \kappa \lambda|f|^{2}, S_{\tilde{C}}\right) \\
& \geqslant|\lambda| \int_{\Omega} \kappa|f|^{2} /\left(\sqrt{2} \kappa_{1}\right) \\
& \geqslant|\lambda| \cdot \kappa_{0} /\left(\sqrt{2} \kappa_{1}\right),
\end{aligned}
$$

where $C \in\left\{A, A_{\omega}\right\}, \tilde{C} \in\left\{\tilde{A}, \tilde{A}_{\omega}\right\}, f \in D(\varepsilon, t) \cap \partial \mathbb{B}(0,1)_{L_{2}(\Omega)}$ and $|\arg (\lambda)| \leqslant 3 \pi / 4$ or $\lambda=0$. Thus, letting $M_{0}:=\sqrt{2} \kappa_{1} / \kappa_{0}$,

$$
\left\|(\lambda-C)^{-1}\right\|_{\mathcal{L}\left(L_{2}(\Omega)\right)} \leqslant M_{0} /|\lambda|, \quad|\arg \lambda| \in(0,3 \pi / 4],
$$


uniformly in $\omega>0$ sufficiently small (invoking the proof of Theorem 3.9 in [18] and well known facts concerning the Robin-Laplacian, it is easy too see that the inverse actually exists). Moreover, if $|\lambda| \leqslant \frac{\omega_{0}}{3 \max \left\{\kappa_{1}, 1\right\}}$, we actually have $\operatorname{dist}\left(\int_{\Omega} \kappa \lambda|f|^{2}, S_{\tilde{C}}\right) \geqslant \omega_{0} / 3+|\lambda|$. If $|\lambda| \geqslant$ $\frac{\omega_{0}}{3 \max \left\{\kappa_{1}, 1\right\}}$, choosing $\omega \leqslant \frac{\omega_{0}}{24 \max \left\{\kappa_{1}, 1\right\}}$, we find $|\lambda| \geqslant 8 \omega$, and a direct computation shows that $4|\lambda+\omega| \geqslant|\lambda|+\omega$. Summarizing, there are numbers $M_{1}>0, m_{0}>0$ and $\theta \in(0, \pi / 2)$ such that

$$
\left\|(\lambda-[A(\varepsilon, t)+(\omega / 2)])^{-1}\right\|_{\mathcal{L}\left(L_{2}(\Omega)\right)} \leqslant M_{1} /\left(|\lambda|+m_{0}\right),
$$

$|\arg \lambda|<\pi / 2+\theta$ or $\lambda=0,(t, \varepsilon) \in\left[0, T^{*}\right] \times\left(0, \varepsilon_{0}\right]$. Since

$$
A(\varepsilon, t)(\lambda-A(\varepsilon, t))^{-1}=\lambda(\lambda-A(\varepsilon, t))^{-1}-1,
$$

we deduce from Theorem 6.9 and the methods developed in part 1 [11],

$$
\left\|\left(u_{\varepsilon}-y\right)(t)\right\|_{L_{2}(\Omega)} \leqslant c \cdot\left[e^{-\mu t / \varepsilon}+\varepsilon \cdot \int_{0}^{\infty} e^{-\mu s} d s \cdot\left\|R\left(u_{\varepsilon}, \rho_{\varepsilon}\right)-\partial_{t} y\right\|_{C\left(\left[0, T^{*}\right], L_{2}(\Omega)\right)} d s\right],
$$

$t \in\left(0, T^{*}\right], \mu>0$. By arranging the regularities via (4.4) using embedding theorems and interpolation we find that for a given $\delta \in\left(0, t^{*}\right]$ we actually have $\left(u_{\varepsilon}-y(\varepsilon)\right) \rightarrow 0$ in $C\left(\left[\delta, T^{*}\right], h^{2+\beta}(\bar{\Omega})\right)$. Of course we also have that $y(\varepsilon) \rightarrow-T(\sigma) H(\sigma):=y(\sigma)$ in $C\left(\left[0, T^{*}\right], h^{3+\beta}(\bar{\Omega})\right)$, hence, $\left(u_{\varepsilon}-y(\sigma)\right) \rightarrow 0$ in $C\left(\left[\delta, T^{*}\right], h^{2+\beta}(\bar{\Omega})\right)$. Finally, from the variation of constants formula we infer that $(\sigma, y(\sigma))$ is a mild solution of $\dot{\tau}=L_{\tau}(H(\tau)+y(\tau))$ $\left(\tau(0)=\rho_{0}, y(\tau)\right.$ being defined in the obvious way), thus, by the regularities, a classical one. The uniqueness of the solution of the quasistationary problem is proven in [10]. The regularity of the limit is obvious.

\section{Conclusions}

In order to justify the quasistationary approximation of a problem involving two coupled evolutions, the following was essential for our analysis:

- Parabolicity of the 'fast evolution'. When considering the fast evolving quantity as a function of the other unknowns, parabolic maximum principles and regularization have been used to derive a priori estimates. These estimates in particular make sure that the solutions of the one parameter family of fast evolutions live on a uniform interval of existence. Roughly speaking, the problems become 'more elliptic' the faster the evolution gets. This reflects in friendly properties of the corresponding (non-local in time) solution operators.

- Spectral properties of the limit problem. For the Stefan problem we essentially used that the spectrum of the Robin-Laplacian is bounded away from zero. In the case of the osmosis problem the situation seems more uncomfortable, as the limit problem for the fast evolution (at a fixed instant of time) has a nontrivial kernel (cf. (2.4)). The conservation of solute (2.3) eliminates this problem.

These observations also apply to the problems considered in part one [11]. 


\section{Appendix}

\subsection{Function spaces}

Let $N \geqslant 2, p>\max \left\{N+2, \frac{N}{1-\alpha}\right\}$ and $X$ be a Banach space. If $U \subset \mathbb{R}^{l}(l \in \mathbb{N})$ is an open set, let $B U C(U, X)$ be the Banach space of all bounded and uniformly continuous $X$-valued functions on $U$. The space $B U C^{k}(U, X)$ contains those elements of $B U C(U, X)$ that possess bounded and uniformly continuous derivatives up to order $k \in \mathbb{N}$. For $k \in \mathbb{N} \cup\{0\}$ and $s \in(0,1), h^{k+s}(\bar{U}, X)$ denotes the little Hölder space, see [16] for a precise definition and basic properties. If $U$ is a domain with sufficiently regular boundary, then $h^{k+s}(\bar{U}, X)$ is known to be the closure of the smooth functions in the usual Hölder space $B U C^{k+s}(U, X)$. All these spaces are given their natural topologies. We shall also use the notation $C^{k+s}(\bar{U}, X):=B U C^{k+s}(U, X)$. A uniformly continuous function defined on a bounded open set $O$ is always identified with its unique extension onto $\bar{O}$ without further mentioning.

If $\sigma \geqslant 0$, we denominate by $W_{p}^{\sigma}(U, X)$ the scale of fractional Sobolev spaces, see [12] for a precise definition. For $\sigma \in(0,1)$ these spaces are equipped with the intrinsic norm $\|u\|_{L_{p}(U, X)}+$ $[u]_{U ; p, \sigma ; X}$, where

$$
[u]_{U ; p, \sigma ; X}:=\left(\int_{U} \int_{U} \frac{\|u(t)-u(s)\|_{X}^{p}}{|t-s|^{l+\sigma p}} d t d s\right)^{1 / p}
$$

If $U=(0, T) \subset \mathbb{R}$, we use the notations $W_{p}^{\sigma}(U, X)=W_{p}^{\sigma}(0, T ; X)$ and $[u]_{U ; p, \sigma ; X}=$ $[u]_{0, T ; p, \sigma ; X}$.

In the case $X=\mathbb{R}$ we write $W_{p}^{\sigma}(U):=W_{p}^{\sigma}(U, \mathbb{R}), h^{k+s}(\bar{U}):=h^{k+s}(\bar{U}, \mathbb{R})$ etc. As usual, function spaces over a manifold are defined by means of a sufficiently smooth atlas.

Let $\Omega, \Gamma, \rho_{0}$ be as specified in Section 3.1. For a perfect interval $J \subset[0, \infty)$ containing 0 we define:

- $E_{1, p}:=W_{p}^{2}(\Omega), E_{0, p}:=L_{p}(\Omega)$;

- $\mathbb{E}_{1, p}(J):=L_{p}\left(\dot{J}, E_{1, p}\right) \cap W_{p}^{1}\left(\dot{J}, E_{0, p}\right)$;

- $\mathbb{E}_{0, p}(J):=L_{p}\left(\dot{J}, E_{0, p}\right)$;

- $\mathbb{F}_{p}(J):=L_{p}\left(\dot{J}, W_{p}^{1-1 / p}(\Gamma)\right) \cap W_{p}^{(1-1 / p) / 2}\left(\dot{J}, L_{p}(\Gamma)\right)$;

- $\mathbb{G}_{p}(J):=\left\{(f, g, x) \in \mathbb{E}_{0, p}(J) \times \mathbb{F}_{p}(J) \times W_{p}^{2-2 / p}(D), B\left(\rho_{0}\right) x=g(0)\right\}$.

In view of standard trace theorems it makes sense to consider subspaces of functions possessing time trace zero:

- ${ }_{0} \mathbb{E}_{1, p}(J):=\left\{f \in \mathbb{E}_{1, p}(J), f(0)=0\right\}$;

- ${ }_{0} \mathbb{G}_{p}(J):=\left\{(f, g, x) \in \mathbb{G}_{p}(J), g(0)=x=0\right\}$

and moreover

- ${ }_{0} \mathbb{E}_{1}(J):=\left\{f \in \mathbb{E}_{1}(J), f(0)=0\right\}$;

- ${ }_{0} \mathbb{G}(J):=\{(f, g, x) \in \mathbb{G}(J), g(0)=x=0\}$,

$\mathbb{E}_{1}(J), \mathbb{G}(J)$ being the spaces defined in Section 3.1. More generally, if $X$ is a given Banach space and $\mathfrak{F} \hookrightarrow B U C(\dot{J}, X)$, we use the notation

$$
{ }_{0} \mathfrak{F}:=\{f \in \mathcal{F} ; f(0)=0\} .
$$

Lemma 6.1 Let $\mathbb{R}^{+}:=[0, \infty)$. If $\lambda>0$ is large enough and $S_{0}:=\left(\partial_{t}+\lambda+A\left(\rho_{0}\right), B\left(\rho_{0}\right), t r_{t=0}\right)$, then

(i) $S_{0} \in \mathcal{L}_{i s}\left(\mathbb{E}_{1, p}\left(\mathbb{R}^{+}\right), \mathbb{G}_{p}\left(\mathbb{R}^{+}\right)\right)$; 
(ii) $S_{0} \in \mathcal{L}_{i s}\left(\mathbb{E}_{1}\left(\mathbb{R}^{+}\right), \mathbb{G}\left(\mathbb{R}^{+}\right)\right)$.

The symbol tr $r_{t=0}$ stands for the time trace mapping at $t=0$.

Proof. Statement (i) is a special case of Theorem 2.3 in [6], cf. also [5]. Statement (ii) can be straightforwardly derived from Theorem 1.4 in [15], estimate (ii) of Theorem 4.4.3 in [16], the representation formula (1.26) in [15] and the observation that we may assume the estimate 3.40 in [17] to be independent of $J$.

Lemma 6.2 Let $X$ be a Banach space, $\sigma \in(0,1), T>0$. Suppose that $Y$ is a Banach space such that $X \cdot Y \hookrightarrow X$ and let

$$
\|x \cdot y\|_{X} \leqslant m \cdot\|x\|_{X}\|y\|_{Y}, \quad m>0, \quad(x, y) \in X \times Y .
$$

Then

(i) $\left\|u^{2}\right\|_{W_{p}^{\sigma}(0, T, X)} \leqslant 4 m \cdot\|u\|_{L_{\infty}(0, T ; Y)}\|u\|_{W_{p}^{\sigma}(0, T ; X)}$

for any $u \in W_{p}^{\sigma}(0, T ; X) \cap L_{\infty}(0, T ; Y)$. More generally, let $b \in{ }_{0} C^{\mu}([0, T], Y)(\sigma<\mu<1)$ satisfy $\|b(t)-b(s)\|_{Y} \leqslant M \cdot|t-s|^{\mu}$. Then

(ii) $[b \cdot u]_{0, T ; p, \sigma ; X} \leqslant C m M \cdot\left(T^{\mu}[u]_{0, T ; p, \sigma ; X}+T^{\mu-\sigma}\|u\|_{L_{p}(0, T ; X)}\right)$,

$C=C(p, \mu, \sigma)$ for any $u \in W_{p}^{\sigma}(0, T ; X)$. In the case $b \in{ }_{0} \operatorname{Lip}([0, T], Y)$ satisfies $\| b(t)-$ $b(s) \|_{Y} \leqslant M \cdot|t-s|$, the same estimate holds true with $\mu=1$.

Proof. For (i) note that

$$
\begin{aligned}
\left(\int_{0}^{T} \int_{0}^{T}\right. & \left.\frac{\left\|u^{2}(t)-u^{2}(s)\right\|_{X}^{p}}{|t-s|^{1+\sigma p}} d t d s\right)^{1 / p} \\
& \leqslant\left(\int_{0}^{T} \int_{0}^{T} \frac{2^{p}\left[\|u(t)[u(t)-u(s)]\|_{X}^{p}+\|u(s)[u(t)-u(s)]\|_{X}^{p}\right]}{|t-s|^{1+\sigma p}} d t d s\right)^{1 / p} \\
& \leqslant 2^{(p+1) / p} \cdot m \cdot\|u\|_{L_{\infty}(0, T ; Y)}\|u\|_{W_{p}^{\sigma}(0, T ; X)} .
\end{aligned}
$$

To see the inequality (ii) we observe that $\|b(t)\|_{Y} \leqslant M \cdot t^{\mu}$. Let $I_{1}(t, s):=\|b(t) \cdot[u(t)-u(s)]\|_{X}$ and $I_{2}(t, s):=\|[b(t)-b(s)] \cdot u(s)\|_{X}$. Then

$$
[b \cdot u]_{0, T ; p, \sigma ; X}^{p} \leqslant \int_{0}^{T} \int_{0}^{T} \frac{I_{1}(t, s)^{p}}{|t-s|^{1+\sigma p}} d t d s+\int_{0}^{T} \int_{0}^{T} \frac{I_{2}(t, s)^{p}}{|t-s|^{1+\sigma p}} d t d s,
$$

and

$$
\int_{0}^{T} \int_{0}^{T} \frac{I_{1}(t, s)^{p}}{|t-s|^{1+\sigma p}} d t d s \leqslant m^{p}\|b\|_{C([0, T], Y)}^{p}\|u\|_{W_{p}^{\sigma}(0, T ; X)}^{p} \leqslant m^{p} M^{p} T^{\mu p}\|u\|_{W_{p}^{\sigma}(0, T ; X)}^{p}
$$

as well as

$$
\begin{aligned}
\int_{0}^{T} \int_{0}^{T} \frac{I_{2}(t, s)^{p}}{|t-s|^{1+\sigma p}} d t d s & \leqslant \int_{0}^{T} \int_{0}^{T} m^{p}\|b\|_{C^{\mu}([0, T], Y)}^{p}|t-s|^{p(\mu-\sigma)-1}\|u(s)\|_{X}^{p} d t d s \\
& \leqslant \frac{(m M)^{p}(2 T)^{p(\mu-\sigma)}}{p(\mu-\sigma)} \int_{0}^{T}\|u(s)\|_{X}^{p} d s .
\end{aligned}
$$




\subsection{Mapping properties of the transformed operators}

Lemma 6.3 There is a ball $B_{a}$ around $\rho_{0}$ in $h^{3+\alpha}(\Gamma)$ such that we have for the mappings $A, B$, $H, L, R$ defined in Section 3.1

- $A(\rho)=\sum_{i, j=1}^{N} a_{i j}(\rho) \partial_{i j}+\sum_{i=1}^{N} a_{i}(\rho) \partial_{i}$, where $\left(a_{i j}, a_{i}\right) \in C^{\infty}\left(B_{a}, h^{2+\alpha}(\bar{\Omega}) \times h^{1+\alpha}(\bar{\Omega})\right)$. Moreover, there exist $0<\Lambda_{0}<\Lambda_{1}$ such that $\Lambda_{0} \leqslant \frac{1}{|\xi|^{2}} \sum_{i, j=1}^{N} a_{i j}(\rho)(x) \xi_{i} \xi_{j} \leqslant \Lambda_{2}, \rho \in B_{a}, x \in \bar{\Omega}, \xi \in \mathbb{R}^{N} \backslash\{0\}$

- $B(\rho) w=\left(\left(b_{1}, \ldots, b_{N}\right)(\rho) \mid \nabla w\right), w \in W_{p}^{1}(\Omega)$, where $b_{i} \in C^{\infty}\left(B_{a}, h^{2+\alpha}(\Gamma)\right)$. Moreover, there exist $0<\lambda_{0}<\lambda_{1}$ such that $\lambda_{0} \leqslant\left(\left(b_{1}, \ldots, b_{N}\right)(\rho)(x) \mid v_{\Gamma}(x)\right) \leqslant \lambda_{2}, \rho \in B_{a}, x \in \Gamma\left(v_{\Gamma}\right.$ being the outer unit normal field on $\Gamma)$;

- $\rho \mapsto L_{\rho} \in C^{\infty}\left(B_{a}, h^{2+\alpha}(\Gamma)\right)$ and there exist $0<l_{0}<l_{1}$ such that $l_{0} \leqslant L_{\rho}(x) \leqslant l_{1}, \rho \in B_{a}$, $x \in \Gamma$;

- $H(\rho)=P(\rho) \rho+Q(\rho)$, and for $(k, v, \tilde{P}) \in\{0,1,2\} \times(0, \alpha] \times\{P, L P\}$ we have $-\tilde{P} \in C^{\infty}\left(B_{a}, \mathcal{H}\left(h^{k+2+v}(\Gamma), h^{k+v}(\Gamma)\right)\right.$ and $Q \in C^{\infty}\left(B_{a}, h^{2+v}(\Gamma)\right)$ (H denoting negative generators of analytic semigroups w.r.t. the specified spaces);

- The mappings $A, B, L, P, Q$ are uniformly Lipschitz continuous on $B_{a}$ w.r.t. the target spaces listed above;

- $R \in C^{\infty}\left(B_{a} \times h^{k+1+v}(\bar{\Omega}), h^{k+v}(\bar{\Omega})\right)(v \in(0, \alpha])$ and there exists $c>0$ such that $\|R(u, \rho)\|_{h^{k+v}(\bar{\Omega})} \leqslant c \cdot\left(1+\|u\|_{h^{k+v}(\bar{\Omega})}\right)\|u\|_{h^{k+1+v}(\bar{\Omega})}\left(\rho \in B_{a}, u \in h^{k+1+v}(\bar{\Omega}), k \in\right.$ $\{0,1\})$;

$\|R(u, \rho)\|_{L_{p}(\Omega)} \leqslant c \cdot\left(1+\|u\|_{C(\bar{\Omega})}\right)\|u\|_{W_{p}^{1}(\Omega)}\left(\rho \in B_{a}, u \in W_{p}^{1}(\Omega)\right)$.

Proof. These properties can be considered as well known [7], [10], [12].

As in Section 3.1 we write $J_{T}:=[0, T]$.

Lemma 6.4 Given $M>\left\|\rho_{0}\right\|_{h^{4+\beta}(\Gamma)}$ there is a $T^{*}>0$ such that for all $T \in\left(0, T^{*}\right]$ we have

$$
\bigcup_{\rho \in V_{M}\left(J_{T}\right)} \rho[[0, T]] \subset B_{a} .
$$

Proof. We have

$$
\begin{aligned}
\|\rho(t)-\rho(0)\|_{h^{3+\alpha}(\Gamma)} & \leqslant\|\rho(t)-\rho(0)\|_{h^{4+\beta}(\Gamma)}^{(1+\beta-\alpha) / 2}\|\rho(t)-\rho(0)\|_{h^{2+\beta}(\Gamma)}^{(1+\alpha-\beta) / 2} \\
& \leqslant(2 M)^{(1+\beta-\alpha) / 2} T^{(1+\alpha-\beta) / 2} .
\end{aligned}
$$

Lemma 6.5 Let $\delta>0, M>\left\|\rho_{0}\right\|_{h^{4+\beta}(\Gamma)}$ be given. There exist $T^{*}, \varepsilon_{0}>0$ such that for all $(T, \varepsilon) \in\left(0, T^{*}\right] \times\left(0, \varepsilon_{0}\right]$ and $\rho \in V_{M}\left(J_{T}\right)$ we have (letting $\rho_{\varepsilon}(t):=\rho(\varepsilon t)$ and $\left.J_{\varepsilon}:=J_{T / \varepsilon}\right)$

(i) (1) $\left\|A\left(\rho_{\varepsilon}\right)-A\left(\rho_{0}\right)\right\|_{\mathcal{L}\left({ }_{0} \mathbb{E}_{1, p}\left(J_{\varepsilon}\right), \mathbb{E}_{0, p}\left(J_{\varepsilon}\right)\right)}<\delta$;

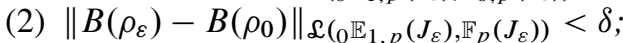

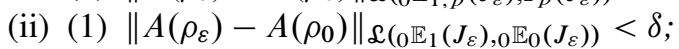

(2) $\left\|B\left(\rho_{\varepsilon}\right)-B\left(\rho_{0}\right)\right\|_{\mathcal{L}\left({ }_{0} \mathbb{E}_{1}\left(J_{\varepsilon}\right),{ }_{0} \mathbb{F}\left(J_{\varepsilon}\right)\right)}<\delta$;

for any $T<T^{*}$ and $\varepsilon<\varepsilon_{0}$.

Proof. In the sequel the symbol $c$ is reserved to denote a generic constant independent of $(T, \varepsilon) \in$ $\left(0, T^{*}\right] \times\left(0, \varepsilon_{0}\right], \rho \in V_{M}\left(J_{T}\right)$ and the number $M$. We restrict ourselves to the most delicate case 
(i) 2): Let $r:=1 / 2-1 /(2 p)$ and $v \in{ }_{0} \mathbb{E}_{1, p}\left(J_{\varepsilon}\right)$. We wright the boundary operator $B$ in the form $B(\sigma) v:=\left(b_{1}, \ldots, b_{N}\right) \cdot \nabla v$, where $b_{j} \in C^{\infty}\left(B_{a}, h^{2, \alpha}(\Gamma)\right)$ according to Lemma 6.3, 6.4. We find

$$
\begin{aligned}
\left\|\left[B\left(\rho_{\varepsilon}\right)-B\left(\rho_{0}\right)\right] v\right\|_{\mathbb{F}_{p}\left(J_{\varepsilon}\right)} & =\left\|\left[B\left(\rho_{\varepsilon}\right)-B\left(\rho_{0}\right)\right] v\right\|_{L_{p}\left(J_{\varepsilon}, W_{p}^{1-1 / p}(\Gamma)\right)} \\
& +\left\|\left[B\left(\rho_{\varepsilon}\right)-B\left(\rho_{0}\right)\right] v\right\|_{W_{p}^{r}\left(J_{\varepsilon}, L_{p}(\Gamma)\right)} \\
= & N_{1}+N_{2}
\end{aligned}
$$

and exemplify the estimate for $N_{2}$ : let $i \in\{1, \ldots, N\}, v_{i}:=\partial_{i} v$ and $b_{\varepsilon}:=b_{i}\left(\rho_{\varepsilon}\right)-b_{i}\left(\rho_{0}\right)$. Then $b_{\varepsilon}(0)=0$ and $\left\|b_{\varepsilon}(t)-b_{\varepsilon}(s)\right\|_{C(\Gamma)} \leqslant c \varepsilon M \cdot|t-s|$. Thus

$$
\begin{aligned}
\int_{(0, T / \varepsilon)^{2}} \frac{\left\|v_{i}(t) b_{\varepsilon}(t)-v_{i}(s) b_{\varepsilon}(s)\right\|_{L_{p}(\Gamma)}^{p}}{|t-s|^{1+r p}} d(t, s) \\
\leqslant \int_{(0, T / \varepsilon)^{2}} \frac{\left\|v_{i}(t)\right\|_{L_{p}(\Gamma)}^{p}\left\|b_{\varepsilon}(t)-b_{\varepsilon}(s)\right\|_{C(\Gamma)}^{p}}{|t-s|^{1+r p}} d(t, s) \\
+\int_{(0, T / \varepsilon)^{2}} \frac{\left\|b_{\varepsilon}(s)\right\|_{C(\Gamma)}^{p}\left\|v_{i}(t)-v_{i}(s)\right\|_{L_{p}(\Gamma)}^{p}}{|t-s|^{1+r p}} d(t, s) .
\end{aligned}
$$

The first summand can be estimated by

$$
\begin{aligned}
& \left\|v_{i}\right\|_{B U C\left(J_{\varepsilon}, L_{p}(\Gamma)\right)}^{p} \cdot c \cdot M^{p} \cdot \varepsilon^{p} \int_{(0, T / \varepsilon)^{2}}|t-s|^{-1+p(1-r)} d(t, s) \\
= & \left\|v_{i}\right\|_{B U C\left(J_{\varepsilon}, L_{p}(\Gamma)\right)}^{p} \cdot c \cdot M^{p} \cdot \varepsilon^{(p-3) / 2} \cdot T^{p(1-r)+1}
\end{aligned}
$$

and (since $p>N+2>3$ ) the desired estimate follows from the embedding ${ }_{0} \mathbb{E}_{1, p}\left(J_{\varepsilon}\right) \hookrightarrow$ $B U C\left(J_{\varepsilon}, W_{p}^{1}(\Gamma)\right)$ (uniformly in $J_{\varepsilon}$, which can be easily seen by extension and restriction).

The second summand is simply estimated by $c \cdot(M T)^{p} \cdot\left[v_{i}\right]_{J_{\varepsilon} ; p, r ; L_{p}(\Gamma)}^{p}$ and the observation that also the estimates characterizing the continuity of the linear mappings

$$
\operatorname{tr}_{\Gamma} \circ \partial_{i}:{ }_{0} \mathbb{E}_{1, p}\left(J_{\varepsilon}\right) \rightarrow{ }_{0} \mathbb{F}_{p}\left(J_{\varepsilon}\right)
$$

are uniform in $J_{\varepsilon}$.

Finally, we mention that the most complicated estimate in the Hölder scale, i.e. the situation in (iii) 2) (and also the other estimates in that scale), can be treated by a similar decomposition of the term $\frac{\left\|v_{i}(t) b_{\varepsilon}(t)-v_{i}(s) b_{\varepsilon}(s)\right\|_{C(\Gamma)}}{|t-s|^{(1+\alpha) / 2}}$ as made above, always making usage of the facts that $\left(B\left(\rho_{\varepsilon}\right)-\right.$ $\left.B\left(\rho_{0}\right)\right)(0)=0$, that the Hölder spaces are Banach algebras w.r.t pointwise multiplication, and that the estimates characterizing the continuity of the linear mapping

$$
\operatorname{tr}_{\Gamma} \circ \partial_{i}: \mathbb{E}_{1}\left(J_{\varepsilon}\right) \rightarrow \mathbb{F}\left(J_{\varepsilon}\right)
$$

are uniform in $J_{\varepsilon}$ (cf. [16], Prop. 1.1.4, 1.1.5 and their proofs in this context).

\subsection{Continuation operators}

Lemma 6.6 (bounded continuation) Let $X$ be a Banach space, $\sigma \in(0,1]$ and $\sigma>1 / p$ such that $W_{p}^{\sigma}(0, T ; X) \hookrightarrow B U C((0, T), X)$ for $T>0$. There exist bounded linear operators 
- $C_{T, X}:{ }_{0} C^{1}([0, T], X) \rightarrow{ }_{0} B U C^{1}\left(\mathbb{R}^{+}, X\right)$;

- $C_{T, X}^{p, \sigma}:{ }_{0} W_{p}^{\sigma}(0, T ; X) \rightarrow{ }_{0} W_{p}^{\sigma}((0, \infty), X)$

such that

- $\left.C_{T, X} f\right|_{[0, T]}=f,\left.C_{T, X}^{p, \sigma} g\right|_{[0, T]}=g$;

- $\sup _{T>0}\left\|C_{T, X}\right\|_{\mathcal{L}\left({ }_{0} C^{1}([0, T], X),{ }_{0} B U C^{1}((0, \infty), X)\right)}<\infty$;

- $\sup _{T>0}\left\|C_{T, X}^{p, \sigma}\right\|_{\mathcal{L}\left(0 W_{p}^{\sigma}(0, T ; X), 0\right.} W_{p}^{\sigma}((0, \infty), X)<\infty$.

Proof. (i) The construction of the operator $C_{T, X}^{p, \sigma}$ as well as all necessary estimates can be taken out of the proof of Proposition 6.1 in [20].

(ii) Given $f \in{ }_{0} C^{1}([0, T], X)$, let $h(x):=-f(-x), x \in[-T, 0]$. Then $h(0)=0, h^{\prime}(0)=f^{\prime}(0)$. Pick $\eta \in \Phi(\mathbb{R})$ such that $\eta=0$ on $(-\infty,-2 T / 3], \eta=1$ on $(-T / 3,0], 0 \leqslant \eta \leqslant 1$, $\left|\eta^{\prime}\right| \leqslant 6 / T$. Define

$$
\bar{h}(x):= \begin{cases}f(x) & \text { if } \quad x \in(0, T] \\ \eta(x) h(x) & \text { if } \quad x \in[-T, 0] \\ 0 & \text { if } \quad x<-T .\end{cases}
$$

Obviously, $\|\bar{h}\|_{B U C((-\infty, 0), X)} \leqslant\|f\|_{B U C((0, T), X)}$. Moreover, as $h(0)=0$ (thus $\left.\|h\|_{B U C((-T, 0), X)} \leqslant T \cdot\left\|f^{\prime}\right\|_{B U C((0, T), X)}\right)$, we find $\left\|\bar{h}^{\prime}\right\|_{B U C((-\infty, 0), X)} \leqslant 7$. $\left\|f^{\prime}\right\|_{B U C((0, T), X)}$. Now. set $\left(C_{T, X} f\right)(y):=\bar{h}(y)$, if $y \leqslant T$ and $\left(C_{T, X} f\right)(T+h):=$ $2 \bar{h}(T)-\bar{h}(T-h)$.

REMARK 6.7 Functions in $L_{p}(0, T ; X)$ are (if necessary) identified with their 0 -extension to the real line without further mentioning. Moreover, the techniques offered in the previous Lemma can obviously be used to construct bounded linear operators $C_{T}:{ }_{0} \mathbb{E}_{1}([0, T]) \rightarrow{ }_{0} \mathbb{E}_{1}\left(\mathbb{R}^{+}\right)$and $C_{T}^{p}$ : ${ }_{0} \mathbb{E}_{1, p}([0, T]) \rightarrow{ }_{0} \mathbb{E}_{1, p}\left(\mathbb{R}^{+}\right)$such that

- $\left.C_{T} f\right|_{[0, T]}=f,\left.C_{T}^{p} g\right|_{[0, T]}=g$;

- $\sup _{T>0}\left\|C_{T}\right\|_{\mathcal{L}\left(0 \mathbb{E}_{1}([0, T]), 0 \mathbb{E}_{1}\left(\mathbb{R}^{+}\right)\right)}<\infty$

- $\sup _{T>0}\left\|C_{T}^{p}\right\|_{\left.\mathcal{L}_{(0} \mathbb{E}_{1, p}([0, T]), 0 \mathbb{E}_{1, p}\left(\mathbb{R}^{+}\right)\right)}<\infty$.

REMARK 6.8 Let $\gamma \in(0,1)$ and $X$ be a Banach space. Clearly, we can extend any function $u \in h^{\gamma}([0, T], X)$ by setting $u(t):=u(T), t>T$, to a function $u_{\infty} \in h^{\gamma}\left(\mathbb{R}^{+}, X\right)$ and obtain $\left\|u_{\infty}\right\|_{h^{\nu}\left(\mathbb{R}^{+}, X\right)}=\|u\|_{h^{\nu}([0, T], X)}$. We denote such an extended function $u_{\infty}$ again by $u$ without further explanation. Obviously, an analogue result holds true in the case of a Lipschitz continuous function. Moreover, for $u \in \operatorname{Lip}([0, T], X)$ and $u_{\varepsilon}(t):=u_{\infty}(\varepsilon t)$ we have for $\gamma \in(0,1]$ that

$$
\left[u_{\varepsilon}\right]_{\mathbb{R}^{+}, \gamma, X} \leqslant \varepsilon^{\gamma} T^{1-\gamma}\left[u_{\infty}\right]_{\mathbb{R}^{+}, \gamma, X}=\varepsilon^{\gamma} T^{1-\gamma}[u]_{[0, T], \gamma, X},
$$

where $[w]_{U, \gamma, X}:=\sup _{s, t \in U} \frac{\|w(t)-w(s)\|_{X}}{|t-s|^{\gamma}}$.

We close the appendix with a 'scaled version' of Theorem IV 2.3.2 in [2]:

Theorem 6.9 Let the assumption IV (2.2.3) in [2] be satisfied and assume additionally that $J$ is finite and assumption III (2.2.1) holds uniformly for $A(s): E_{1}(s) \rightarrow E_{0}(s \in J)$. Then, if $U_{\varepsilon}(t, s)=U_{\varepsilon}(t, s)(A)$ is the evolution system associated to the $A_{\varepsilon}(s):=(1 / \varepsilon) A(s)(A \in Q)$, we have for some positive numbers $\mu_{0}, \mu_{1}, \varepsilon_{0}$ that

$$
\left\|U_{\varepsilon}(t, s)\right\|_{\mathcal{L}\left(E_{0}\right)} \leqslant \mu_{0} \cdot e^{-\mu_{1}(t-s) / \varepsilon}, \quad s \in J, A \in Q,(t, s) \in J_{\Delta}^{*}, 0<\varepsilon \leqslant \varepsilon_{0} .
$$


Proof. By $e^{-t A(s)}$ we denote the strongly continuous analytic semigroup generated by $-A(s)$.

Observe that for $\alpha, \varepsilon>0$ we have $[(1 / \varepsilon) A(s)]^{\alpha}=\varepsilon^{-\alpha} A(s)^{\alpha}$ as well as $e^{-t((1 / \varepsilon) A(s))}=$ $e^{(-t / \varepsilon) A(s)}(s \in J)$. Formula IV (2.2.7), Lemma III 2.2.1 in [2] and (the proof of) Theorem 2.6.13 c) in [18] imply that we can diversify the arguments used to prove IV (2.3.20) via formula IV (2.3.19) in [2] in a fashion oriented at Section 6 in [11] to obtain (simply writing $A_{\varepsilon}$ instead of $(1 / \varepsilon) A(s)$ as well as $a_{\varepsilon}:=a_{A_{\varepsilon}}, k_{\varepsilon}:=k_{A_{\varepsilon}}$ etc.)

$$
\begin{aligned}
\left\|a_{\varepsilon}^{0}\right\|_{\mathcal{L}\left(E_{0}\right)} & \leqslant c_{0} \cdot e^{-c_{1}(t-s) / \varepsilon} \\
\left\|a_{\varepsilon}\right\|_{\mathcal{L}\left(E_{0}\right)} & \leqslant c_{0} \cdot(t-s)^{\alpha-1} \cdot e^{-c_{1}(t-s) / \varepsilon} \\
\left\|k_{\varepsilon}^{0}\right\|_{\mathcal{L}\left(E_{0}\right)} & \leqslant c_{0} \cdot \varepsilon^{1-\alpha} \cdot(t-s)^{\rho-1} \cdot e^{-c_{1}(t-s) / \varepsilon} \\
\left\|k_{\varepsilon}\right\|_{\mathcal{L}\left(E_{0}\right)} & \leqslant c_{0} \cdot \varepsilon^{1-\alpha} \cdot(t-s)^{\rho+\alpha-2} \cdot e^{-c_{1}(t-s) / \varepsilon} \\
\left\|w_{\varepsilon}^{0}\right\|_{\mathcal{L}\left(E_{0}\right)} & \leqslant c_{0} \cdot \varepsilon^{1-\alpha} \cdot(t-s)^{\rho-1} \cdot e^{-c_{1}(t-s) / \varepsilon} \\
\left\|w_{\varepsilon}\right\|_{\mathcal{L}\left(E_{0}\right)} & \leqslant c_{0} \cdot \varepsilon^{1-\alpha} \cdot(t-s)^{\rho+\alpha-2} \cdot e^{-c_{1}(t-s) / \varepsilon}
\end{aligned}
$$

where $\alpha$ comes now from IV (2.3.5) [2] and $c_{0}, c_{1}$ are independent of $s \in J, A \in Q$ and $\varepsilon<\varepsilon_{0}$ cf. [11], appendix. The assertion follows from formula IV (2.3.19) in [2]. The assumption that $J$ should be finite enters in the estimate corresponding to IV (2.3.15) [2].

Acknowledgement. The author gratefully acknowledges the financial support of the German Science Foundation (DFG) within the Priority Program (SPP) 1506 "Transport Processes at Fluidic Interfaces".

\section{References}

1. Amann, H., Existence and Regularity for Semilinear Parabolic Evolution Equations, Annali della Scuola Normale Superiore di Pisa - Classe di Scienze (1984) Vol. 11, Is. 4, 593-676. Zb10625. 35045 MR0808425

2. Amann, H., Linear and Quasilinear Parabolic Problems, Birkhäuser, Basel, 1995. Zb10819.35001 MR1345385

3. Boulkhemair, A. \& Chakib, A.: On the uniform Poincaré inequality. Comm. Partial Differential Equations 32 (2007), 1439-1447. Zbl1128.26010 MR2354499

4. Bergner, M., Escher, J. \& Lippoth, F., A blow-up criterion for surface evolution models. Nonlinear Analysis, T, M\&A 75 (2012), 3951-3963.

5. Denk, R., Hieber, M. \& Prüß, J., Optimal $L_{p}-L_{q}$-regularity for parabolic problems with inhomogeneous boundary data. Math. Z. 257 (2007), 193-224. Zbl1210. 35066 MR2318575

6. Denk, R., Prüß, J. \& Zacher, R., Maximal $L_{p}$-regularity of parabolic problems with boundary dynamics of relaxation type. Journ. Funct. Anal. 255 (2008), 3149-3187. Zb11160 . 35030 MR2464573

7. Escher, J., Classical solutions for an elliptic parabolic system. Interfaces and Free Boundaries 6 (2004), 175-193. Zbl1057 . 35097 MR2079602

8. Escher, J. \& Simonett, G., Classical solutions for Hele-Shaw models with surface tension. Adv. Differential Equations 2 (1997), 619-642. Zbl1023. 35527 MR1441859

9. Frolova, E. V. \& Solonnikov, V. A., Justification of a quasistationary approximation for the Stefan problem. Journal of Mathematical Sciences 152 (2008), 741-768.

10. Kneisel, C.-H., Über das Stefan-Problem mit Oberfächenspannung und thermischer Unterkühlung. VDM Verlag Dr. Müller, 2008 
11. Lippoth, F., On the justification of the quasistationary approximation of several parabolic moving boundary problems - Part I. Nonlinear Analysis: Real World Applications 17 (2014), 1-22. Zbl1296. 35221 MR3158456

12. Lippoth, F. \& Prokert, G., Classical solutions for a one-phase osmosis model. Journal of Evolution Equations 12 (2012), 413-434. Zbl1254. 35250 MR2923941

13. Lippoth, F. \& Prokert, G., Stability of equilibria for a two-phase osmosis model. NoDEA 21 (2014), 129-148. Zbl1301.35223 MR3158804

14. Lippoth, F., Peletier, M. \& Prokert, G., A moving boundary problem for the Stokes equations involving osmosis: variational modelling and short-time well-posedness, European J. Appl. Math. 27 (2016), 647666. MR3517540

15. Lunardi, A., Maximal space regularity in inhomogeneous initial boundary value parabolic problems. Num. Funct. Anal. and Opt. 10 (1989), 323-349 Zb10653. 35047 MR0989538

16. Lunardi, A., Analytic Semigroups and Optimal Regularity in Parabolic Problems. Birkhäuser, Basel, 1995 Zb10816.35001 MR1329547

17. Lunardi, A., Sinestrari, E. \& v. Wahl, W., A semigroup approach to the time dependent parabolic initialboundary value problem. Differential and Integral Equations 5 (1992), 1275-1306. Zbl0758.34048 MR1184027

18. Pazy, A., Semigroups of Linear Operators and Applications to Partial Differential Equations. Springer, New York, 1983 Zb10516.47023 MR0710486

19. Pickard, W. F., Modelling the Swelling Assay for Aquaporin Expression. J. Math. Biol. 57 (2008), 883903. Zbl1161.92023 MR2439677

20. Prüss, J., Saal, J. \& Simonett, G., Existence of analytic solutions for the classical Stefan problem. Math. Ann. 338 (2007), 703-755. Zbl1130. 35136 MR2317935

21. Prüss, J., Simonett, G. \& Zacher, R., Qualitative behavior of solutions for thermodynamically consistent Stefan problems with surface tension. Arch. Ration. Mech. Anal. 207 (2012), 611-667. Zbl1269. 80004 MR3005325

22. Triebel, H., Theory of function spaces. Birkhäuser, Basel, 1983 Zbl0546. 46028 MR0781540

23. Solonnikov, V. A., On the justification of the quasistationary approximation in the problem of motion of a viscous capillary drop. Interfaces and Free Boundaries 1, (1999), 125-173. Zb10974. 35097 MR1867129

24. Verkman, A. S., Water channels in cell membranes. Annu. Rev. Physiol. 54 (1992), 97-108.

25. Verkman, A. S., Solute and makromolecular diffusion in cellular aqueous compartments. Trends Biochem. Sci. 23 (2000), 27-33.

26. Van Moers, P., Osmotic cell swelling in the fast diffusion limit. MSc thesis, Technical University of Eindhoven, 2011

27. Yi, F., Asymptotic behaviour of the solutions of the supercooled Stefan problem. Proceedings of the Royal Society of Edinburgh 127 A (1997), 181-190. Zbl0876. 35135 MR1433091

28. Zaal, M., Linear Stability of Osmotic Cell Swelling. MSc thesis, Vrije Universiteit Amsterdam, 2008

29. Zaal, M., Cell swelling by osmosis: A variational approach. Interfaces and Free Boundaries 14 (2012), 487-520.

30. Zaal, M., Cell swelling by osmosis: Existence, uniqueness and long-time behaviour. Mathematical Methods in the Applied Sciences 38 (2015), 380-392. 